\title{
Stability of a Compound Sessile Drop at the Axisymmetric Configuration
}

Ying Zhang, ${ }^{1,2}$ Dominique Chatain, ${ }^{3}$ Shelley L. Anna, ${ }^{2,4,5 a}$ and Stephen Garoff ${ }^{1,2}$

${ }^{1}$ Physics Department, Carnegie Mellon University, Pittsburgh, PA 15213, USA

${ }^{2}$ Center for Complex Fluids Engineering, Carnegie Mellon University, Pittsburgh, PA 15213,

USA

${ }^{3}$ Aix-Marseille Université, CNRS, CINaM UMR 7325, 13288 Marseille, France

${ }^{4}$ Chemical Engineering Department, Carnegie Mellon University, Pittsburgh, PA 15213, USA

${ }^{5}$ Mechanical Engineering Department, Carnegie Mellon University, Pittsburgh, PA 15213, USA

\begin{abstract}
The equilibrium configuration of compound sessile drops has been calculated previously in the absence of gravity. Using the Laplace equations, we establish seven dimensionless parameters describing the axisymmetric configuration in the presence of gravity. The equilibrium axisymmetric configuration can be either stable or unstable depending on the fluid properties. A stability criterion is established by calculating forces on a perturbed Laplacian shape. In the zero Bond number limit, the stability criterion depends on the density ratio, two ratios of interfacial tensions, the volume ratio of the two drops, and the contact angle. We use Surface Evolver to examine the stability of compound sessile drops at small and large Bond numbers and compare with the zero Bond number approximation. Experimentally, we realize a stable axisymmetric compound sessile drop in air, where the buoyancy force exerted by the air is negligible. Finally, using a pair of fluids in which the density ratio can be tuned nearly independently of the

a Author to whom correspondence should be addressed. Electronic mail: sanna@cmu.edu
\end{abstract}


interfacial tensions, the stability transition is verified for the axisymmetric configuration. Even though the perturbations are different for the theory, simulations and experiments, both simulations and experiments agree closely with the zero Bond number approximation, exhibiting a small discrepancy at large Bond number.

\section{Keywords}

Compound drop; Sessile drop; Surface tension; Interfacial tension; Bond number; Partially miscible fluids

\section{Introduction}

Surface tension plays a key role in the flotation mechanism of very small objects at liquid interfaces $^{1-3}$, especially for dense objects floating on a less dense fluid phase. The mechanical equilibrium of solid particles at a gas/liquid interface has been analyzed comprehensively using a variational approach ${ }^{4}$. While the configuration of solid particles at a gas/liquid interface is determined by Young's contact angle, the configuration of a sessile drop at a liquid/liquid interface is determined by Neumann's triangle ${ }^{5-7}$ which says that the net force per unit length between the three phases must be zero. While dense solid objects have been observed to float on liquids $^{1-3}$, relatively few observations have been reported of dense drops floating on a lighter liquid. Phan $e t a l^{8,9}$ reported that dense drops can float on less dense fluids for two water-oil fluid pairs through the distortion of the fluid interface.

Reducing the lower fluid phase from an infinite reservoir to a finite-sized drop breaks the

translational symmetry, resulting in a different geometry of the compound sessile drop ${ }^{10-12}$. The configuration becomes that of two immiscible drops in contact with one another, on top of a solid 
substrate, and submerged in an ambient external fluid phase such as air or another fluid. The lower liquid phase contacts the solid substrate with a fixed static contact angle, while the shapes of the three fluid interfaces formed by contact between each fluid pair are determined by Neumann's triangle and the Laplace equations balancing surface tension and gravity. Here we examine the stability of the equilibrium configuration in which the upper drop is centered at the apex of the lower drop and both drops achieve axisymmetric shapes.

For the compound sessile drop geometry, a stable configuration has not been observed before if both drops are more dense than the ambient phase. Previous reports have established a stable configuration utilizing the buoyancy force induced by the ambient phase. Neeson et al ${ }^{12}$ experimentally realized a compound sessile drop consisting of a tetradecane lens on a perfluorooctane drop in contact with a hydrophobic glass substrate under water. Tetradecane is less dense than the ambient water phase. More generally, it is not known whether fluids that are more dense than the ambient phase (e.g. air) can assume a stable axisymmetric configuration. In the present paper, we report an experimental realization of a stable axisymmetric compound sessile drop of two immiscible liquids in air. By systematically varying the fluid properties, we observe the transition from a stable configuration to an unstable configuration.

Figure 1 shows two typical experimental configurations that result from manually depositing one smaller drop $(2 \mu \mathrm{L})$ on the top of a large sessile drop $(50 \mu \mathrm{L})$. In the two cases shown, the surface and interfacial tensions are very similar but the density ratios are different. Figure 1a shows an axisymmetric configuration that is stable against small perturbations from the apex position. The ratio of the density of the upper drop to the density of the lower drop is 0.859 . The 


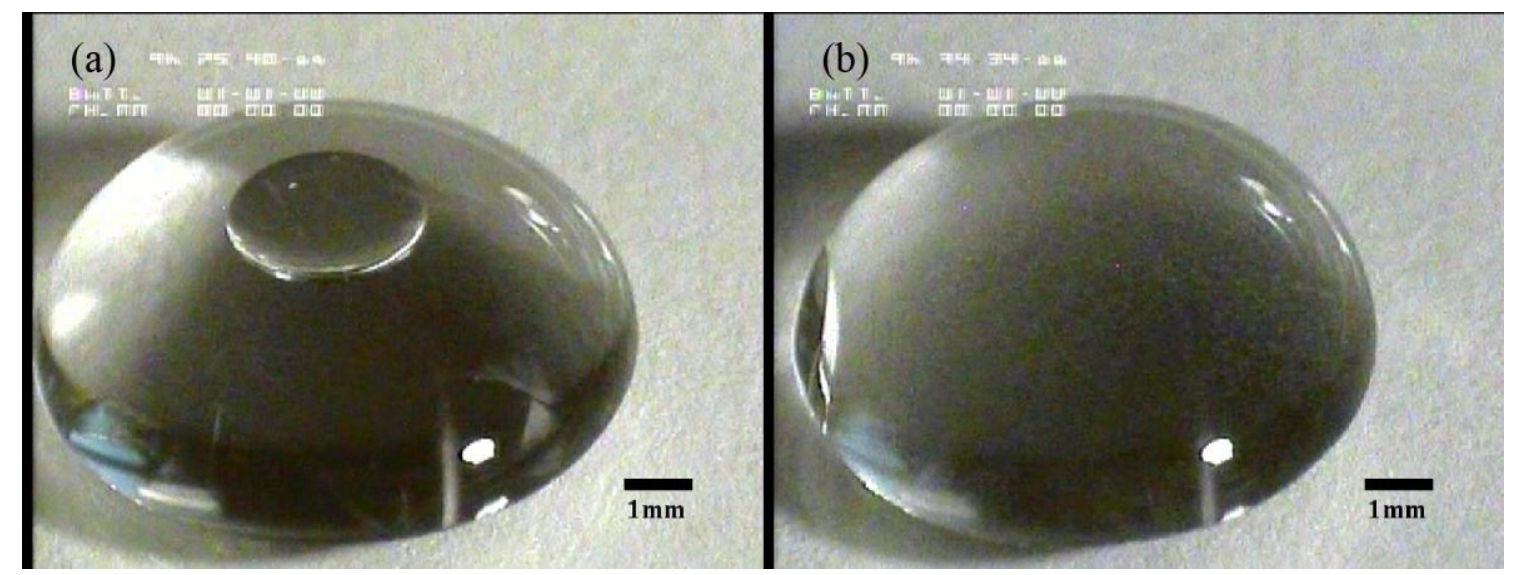

Figure 1. (a) Stable axisymmetric configuration using two liquid phases obtained from a phase separated mixure of aqueous $22 \% \mathrm{CsCl}$ solution with benzyl alcohol, density ratio 0.859. The stability of the configuration subject to perturbations can be seen in Supplementary Information Video 1. (b) Janus shape of compound sessile drop using two liquid phases obtained from a phase separated mixture of aqueous $10 \% \mathrm{CsCl}$ solution with benzyl alcohol, density ratio 0.955 . More detail on the fluid properties can be found in the experimental section. The evolution from the unstable axisymmetric configuration to the Janus shape can be viewed in Supplementary Information Video 2.

fluid pair is obtained by mixing $22 \% \mathrm{CsCl}$ in water with benzyl alcohol, and allowing the slightly immiscible system to phase separate into a water-rich phase and a benzyl alcohol rich phase. Video 1 of the Supplementary Materials shows the restoration of the top drop to the axisymmetric position after the top drop is released from a perturbed position. Figure $1 \mathrm{~b}$ shows the Janus-like configuration of the two drops side by side, where both are in contact with the solid substrate. In this case, the two liquid phases are obtained from the phase separated mixture of $10 \% \mathrm{CsCl}$ in water which achieves a density ratio of 0.955 . Video 2 of the Supplementary Materials shows that the axisymmetric configuration is not stable: once the top drop is deposited at the center, it immediately slides off to the side.

The fundamentally different compound sessile drop configurations shown in Figure 1 inspire us to examine the stability criterion for the axisymmetric equilibrium shape, as a function of the 
interfacial tensions and other physical properties. In the following sections we identify the physical conditions needed to obtain an immiscible drop stably situated at the apex of a second sessile drop.

Compound sessile drops can achieve more possible configurations than isolated compound drops found in multiple drop processes ${ }^{13,14}$ as a result of the added solid surface. The axisymmetric configuration and the Janus configuration are two of the possibilities ${ }^{10-12,15}$. The equilibrium shape of the Janus configuration has been found previously using phase field simulations that incorporate the wetting properties ${ }^{15}$. Equilibrium axisymmetric shapes have been examined in the absence of gravity ${ }^{11,12}$.

More generally, the shapes of the interfaces in compound sessile drops are determined by the balance of surface tension and gravity, which can be assessed by the magnitude of the Bond number, defined as $B o=\Delta \rho g L^{2} / \gamma$, where $\Delta \rho$ is the density difference between the liquid in the drop of interest and the ambient fluid, $L=V^{1 / 3}$ is the characteristic length of the drop estimated from the drop volume $V$, and $\gamma$ is the interfacial tension between the drop and the ambient phase. Each component of the compound drop will have a different balance of these forces, assessed by the relevant Bond number for each component. In the limit of small Bond number, the shape deviation due to gravity is negligible, and the interface shape can be approximated as a spherical cap. This approximation permits analytical solutions to the compound drop configuration ${ }^{16,17}$.

In the present paper we consider the stability of the axisymmetric configuration consisting of a drop sitting at the apex of a second sessile drop in contact with a solid substrate (Figure 1a). Using the Laplace equations, we first determine the dimensionless parameters governing the 
equilibrium shape of the compound sessile drop. Incorporating the force due to gravity in the zero Bond number limit, corresponding to small size drops in which gravity is still present, the stability criterion distinguishing between an energy minimum or maximum is determined. We

use Surface Evolver ${ }^{18-20}$ to simulate fluid systems at small and intermediate Bond numbers and compare the observed stability conditions with the zero Bond number stability criterion. In these cases stability is determined based on the minimization of Gibbs free energy. Finally, we select several experimental fluid systems with small and large interfacial tension to demonstrate both stable and unstable axisymmetric configurations.

\section{Theoretical Considerations}

As illustrated by Berthier et al. using the Steiner symmetrization process ${ }^{21}$, rotationally symmetric configurations of drops or fluid surfaces tend to have lower energy than arbitrary shapes $^{20}$. Even though Steiner symmetrization itself does not produce the minimum energy configuration, it shows that the minimum energy state must be rotationally symmetric ${ }^{20}$. However, Steiner symmetrization does not indicate whether the rotationally symmetric state is an energy minimum (stable) or energy maximum (unstable). This motivates us to ask whether the axisymmetric compound sessile drop configuration is an energy maximum or energy minimum.

\section{Governing parameters for axisymmetric configurations}

We first consider the governing parameters for the equilibrium configuration of an axisymmetric compound sessile drop. As shown schematically in Figure 2, the lower drop, which is in direct contact with the solid substrate, is denoted as Drop 1 while the upper drop is denoted as Drop 2. The ambient phase can be either air or another fluid phase. The center of the basal 


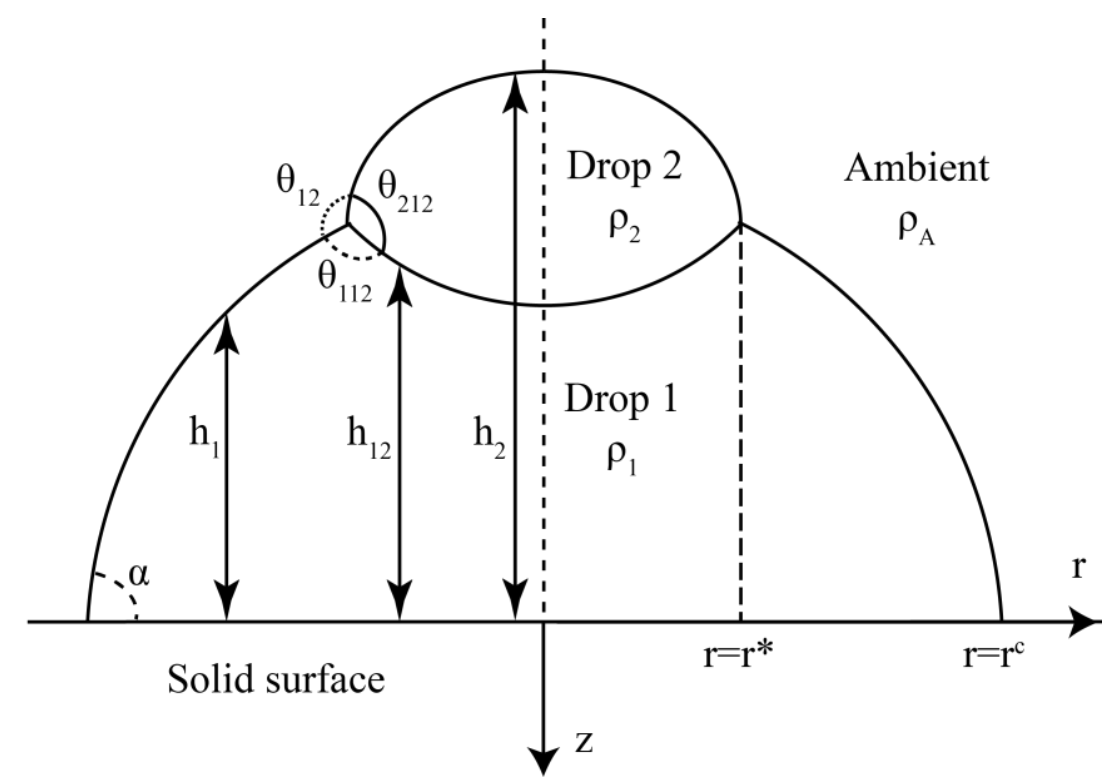

Figure 2. Schematic diagram of axisymmetric compound sessile drop. The lower drop denoted Drop 1 is in direct contact with the solid substrate. The upper drop is denoted as Drop 2. The ambient fluid can be either air or another fluid with density $\rho_{A}$.

plane of the drop is taken as the origin of the coordinate system and the downward vertical direction is defined to be positive. The densities of the three fluid phases are denoted as $\rho_{1}, \rho_{2}$ and $\rho_{\mathrm{A}}$, respectively. The distance from the basal plane to the phase boundary between the ambient phase and Drop 1 is denoted as $h_{1}$; of the distance to the boundary between Drop 2 and the ambient phase is denoted by $h_{2}$; and $h_{12}$ denotes the distance to the boundary between Drop 1 and Drop 2. The first and second order derivatives of the Drop 1 interface with respect to the radius are expressed using simplified notation as $h_{1, r}=\partial h_{1} / \partial r$ and $h_{1, r r}=\partial^{2} h_{1} / \partial r^{2}$, respectively. The derivatives of other variables are defined in the same fashion.

Following the derivation of Pujado and Scriven $^{5}$ outlined for an equilibrium sessile lens between two other infinite fluid phases, the three phase boundaries for the compound sessile drop each satisfy the Laplace equation. 


$$
\begin{aligned}
& \left(\frac{1}{r} \frac{h_{1, r}}{\left(1+h_{1, r}{ }^{2}\right)^{1 / 2}}+\frac{h_{1, r r}}{\left(1+h_{1, r}{ }^{2}\right)^{3 / 2}}\right)-\kappa_{1}^{0}=\frac{\Delta \rho_{1 A} g}{\gamma_{1 A}}\left(h_{1}-h_{1}^{0}\right) \\
& \left(\frac{1}{r} \frac{h_{2, r}}{\left(1+h_{2, r}{ }^{2}\right)^{1 / 2}}+\frac{h_{2, r r}}{\left(1+h_{2, r}{ }^{2}\right)^{3 / 2}}\right)-\kappa_{2}^{0}=\frac{\Delta \rho_{2 A} g}{\gamma_{2 A}}\left(h_{2}-h_{2}^{0}\right), \\
& \left(\frac{1}{r} \frac{h_{12, r}}{\left(1+h_{12, r}{ }^{2}\right)^{1 / 2}}+\frac{h_{12, r r}}{\left(1+h_{12, r}{ }^{2}\right)^{3 / 2}}\right)-\kappa_{12}^{0}=\frac{\Delta \rho_{12} g}{\gamma_{12}}\left(h_{12}-h_{12}^{0}\right)
\end{aligned}
$$

where $\kappa_{1}^{0}, \kappa_{2}^{0}$ and $\kappa_{12}^{0}$ represent the curvature of the interfaces (or extended interfaces) on the symmetry axis at $h_{1}^{0}, h_{2}^{0}$ and $h_{12}^{0}$, the height of each interface along the symmetry axis; $\Delta \rho_{1 A}, \Delta \rho_{2 A}$, and $\Delta \rho_{12}$ represent the density differences of the phases; and $\gamma_{1 A}, \gamma_{2 A}$, and $\gamma_{12}$ are the three interfacial tensions between the three fluid phases. In the remainder of the manuscript, we refer to any interface shape that satisfies the Laplace equation, e.g. Eq. (1a), i.e., that is subject to a balance of gravity and surface tension forces, as a Laplacian shape.

The three fluid phase contact line must satisfy the Neumann's triangle condition at $x=r^{*}$,

$$
\begin{aligned}
& \cos \left(\theta_{12}\right)=-\frac{\gamma_{1 A}^{2}+\gamma_{2 A}^{2}-\gamma_{12}^{2}}{2 \gamma_{1 A} \gamma_{2 A}} \\
& \cos \left(\theta_{112}\right)=-\frac{\gamma_{12}^{2}+\gamma_{1 A}^{2}-\gamma_{2 A}^{2}}{2 \gamma_{12} \gamma_{1 A}},
\end{aligned}
$$

where the angle $\theta_{12}$ is the angle formed by the interfaces $h_{1}$ and $h_{2}$, and $\theta_{112}$ is the angle formed by the interfaces $h_{1}$ and $h_{12}$.

Additionally, the three Laplace equations given in Eq. (1) are subject to the boundary conditions,

$$
\begin{gathered}
h_{2, r}=h_{12, r}=0 \text { at } r=0 \\
h_{1}=h_{2}=h_{12} \text { at } r=r^{*}, \\
h_{1, r}=\tan \alpha \quad \text { at } \mathrm{r}=r^{c} \\
\Delta \rho_{1 A} g h_{1}^{\mathrm{O}}-\gamma_{1 A} \kappa_{1}^{\mathrm{O}}=\Delta \rho_{2 A} g h_{2}^{\mathrm{O}}-\gamma_{2 A} \kappa_{2}^{\mathrm{O}}+\Delta \rho_{12} g h_{12}^{\mathrm{O}}-\gamma_{12} \kappa_{12}^{O} .
\end{gathered}
$$


Here, Eq. (3a) represents three geometric relationships: the symmetry condition about the central axis, contact of the interfaces at the three phase fluid contact line, and the contact angle condition at the solid substrate, respectively. Eq. (3b) constrains the sum of the pressure jumps across each interface to be zero around a closed path to maintain hydrostatic equilibrium ${ }^{5}$. Note that the Laplace equations (Eq.(1)) and boundary conditions (Eq.(3)) only depend on the density differences and the pressure jump due to curvature. This suggests that the ambient density $\rho_{A}$ and the ambient pressure $P_{0}$, can be treated as reference quantities, and the system can be taken to have zero ambient pressure, zero ambient density, and the reduced densities $\rho_{1}-\rho_{A}$ and $\rho_{2}-\rho_{A}$ for Drop 1 and Drop 2 respectively.

The three Laplace equations can be nondimensionalized using a single capillary length $a_{1 A}=\left(\gamma_{1 A} / \Delta \rho_{1 A} g\right)^{1 / 2}$ corresponding to the lower drop. The shape of the compound sessile drop is governed by seven dimensionless parameters, following a similar derivation to that of Pujado and Scriven ${ }^{5}$ for the shape of a sessile lens resting on a bulk fluid phase. Scaled by the lower drop capillary length, the seven dimensionless parameters are: two ratios of interfacial tensions $\gamma_{12} / \gamma_{1 A}$ and $\gamma_{2 A} / \gamma_{1 A}$; two ratios of capillary lengths $a_{2 A} / a_{1 A}$ and $a_{12} / a_{1 A}$; the contact angle on the solid substrate $\alpha$; the Bond number of Drop $1 B Q_{1}=V_{1}^{2 / 3} /\left(\gamma_{1 A} / \Delta q_{A} g\right)$; and the volume ratio of the two drops $V_{2} / V_{1}$.

The differential equations given in Eqs.(1) and (3) do not have a closed-form solution. Furthermore, since the shape of the compound sessile drop is governed by seven parameters, it is difficult for numerical solutions to provide systematic physical insight. Thus, we consider the zero Bond number limit to reduce the number of governing parameters and gain insight into the 
stability of the axisymmetric configuration. In the zero Bond number limit, the parameters governing the shape reduce to four dimensionless parameters: $\gamma_{12} / \gamma_{1 A}, \gamma_{2 A} / \gamma_{1 A}, V_{2} / V_{1}$ and $\alpha$. We further consider cases with fixed contact angle $\alpha$, further reducing the parameter space to three governing dimensionless parameters.

\section{Stability criterion based on Laplacian shapes}

For a single axisymmetric sessile drop at small Bond number, the asymptotic solution to the Laplace equation (Eq. (1)) can be approximated by a spherical cap with first order error in the Bond number ${ }^{22-24}$. The equilibrium profile of the compound sessile drop in the absence of gravity was solved by Mahadevan et $a l^{11}$ and by Neeson et $a l^{12}$ independently, with similar approximations applied in the earlier work of Torza and Mason ${ }^{16}$. However, in determining the stability criteria for the compound sessile drop, neither the forces arising from gravity (the weight of the drop itself) nor buoyancy (displacement of the bottom fluid by the top drop) can be omitted; otherwise, the compound sessile drop is stable at any orientation. The assumption of spherical cap drop shapes is incompatible with the presence of a gravity field ${ }^{25}$. Hydrostatic pressure as a function of height within a drop results in a curvature difference at different heights; therefore, neither the gravity force nor the buoyancy force are defined in spherical cap drops.

In the cases of one sessile ${ }^{26,27}$ or pendant $\operatorname{drop}^{28,29}$ in contact with a solid surface, difficulty arises in stability analysis because the perturbed shape can be any generic function, not necessarily restricted to the family of Laplacian shapes; i.e., perturbation analysis for liquid drops lies in the mathematical formulation of functional analysis, beyond the traditionally better understood realm of energy minimization based on convex analysis ${ }^{30}$. Because of this difficulty, 
(a)

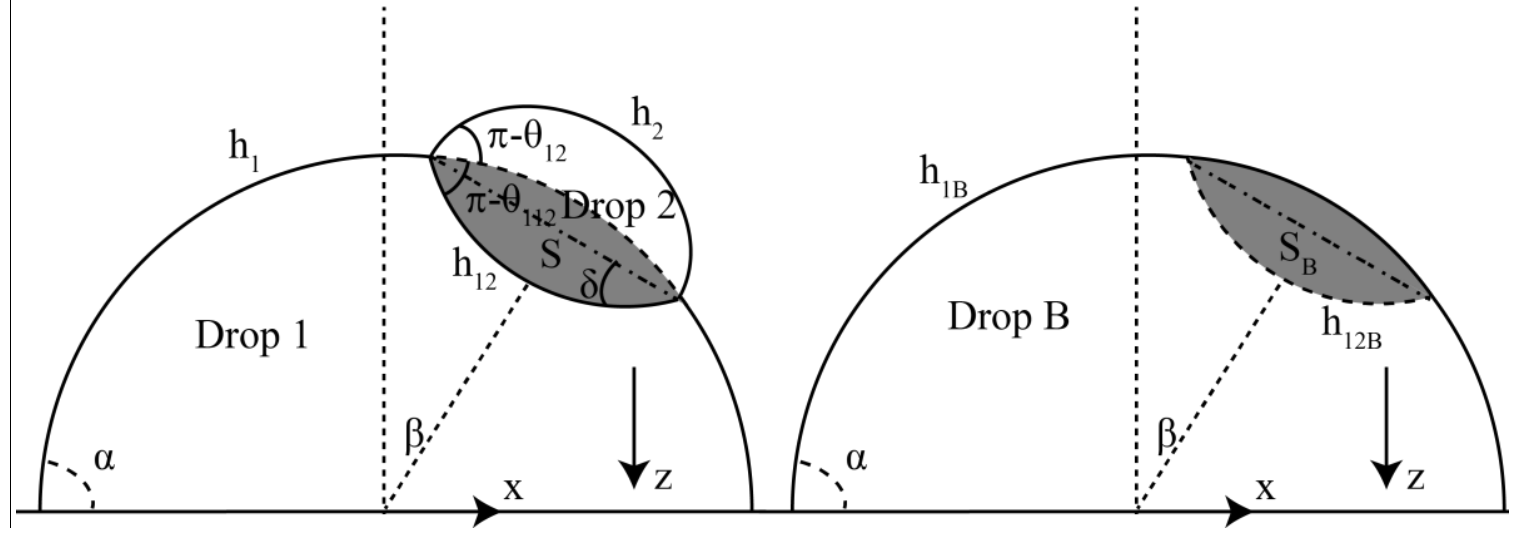

Figure 3. (a) Top drop ( Drop 2) offset from the axisymmetric position. Bottom drop (Drop 1) shape is constrained to a Laplacian shape. The net force exerted on the top drop can be calculated to determine the stability of the axisymmetric equilibrium shape. Shaded region $S$ has volume $V_{S}$. (b) One sessile drop on top of a solid surface with the same shape as Drop 1 in (a). The dashed line $h_{12 \mathrm{~B}}$ is drawn to be identical to the interface $h_{12}$, and the shaded region $S_{B}$ also has volume $V_{S}$.

we limit the stability analysis presented here to the subset of Laplacian drop shapes. In contrast to the typical stability analysis for mechanical equilibrium based on the second derivative of the potential energy at the extremum (or the first derivative of a conservative force $)^{31,32}$, we directly calculate the force on the top drop when it is perturbed away from the axisymmetric position while interfaces are constrained to be of Laplacian shape.

Consider the configuration of a compound sessile drop shown in Figure 3a where the top drop (Drop 2 of Laplacian shape) is perturbed from the axisymmetric position by an angle $\beta$, while the bottom drop (Drop 1) is of continuous Laplacian shape. The extension of the surface of Drop 1 shown by the dashed line and the interface between Drop 1 and Drop $2\left(h_{12}\right)$ encloses a shaded region denoted $S$, with volume denoted $V_{S}$ as shown in Figure 3a. The forces exerted on Drop 2 (enclosed by $h_{12}$ and $h_{2}$ ) include the gravitational force and the forces exerted by Drop 1 through contact, i.e., the pressure force and the surface tension force. The total force exerted 
through contact will be calculated by constructing an identical Laplacian shape of a single sessile drop.

Figure 3b shows a single sessile drop denoted Drop B, which has the identical shape to Drop 1 including the continuation of the surface of Drop 1; thus, the volume of Drop B is equal to the sum of volumes of Drop 1 and the shaded volume, $V_{B}=V_{1}+V_{S}$. In Drop B, the dashed line $h_{12 \mathrm{~B}}$ shown in Figure $3 \mathrm{~b}$ is identical to the interface $h_{12}$ (in Figure $3 \mathrm{a}$ ) to create the identical shaded volume region $S$ in this single sessile drop case. Since both Drop 1 and Drop B have the same Laplacian shape, pressure fields along the interfaces $h_{12}$ and $h_{12 \mathrm{~B}}$ are identical, and the surface tension forces along the three phase contact line (the intersection line of $h_{1}$ and $h_{12}$ and the intersection line of $h_{1 \mathrm{~B}}$ and $h_{12 \mathrm{~B}}$ ) are also identical in the two different configurations. Therefore the force exerted on Drop 2 through contact in the compound drop case is identical to the force exerted on the shaded region $S_{B}$ (Figure $3 b$ ) through contact in the single drop case: $\vec{F}_{2, \text { contact }}=\vec{F}_{S_{B}, \text { contact }}$.

The force exerted on the shaded region through contact in the single drop case can be derived based on the properties of Laplacian shapes ${ }^{25,33}$. Within a drop of Laplacian shape, fluid is in hydrostatic equilibrium i.e., any material body drawn experiences zero net force. The net force on the material body of the shaded region in Figure $3 \mathrm{~b}$ is the sum of the gravitational force and the force through contact: $\vec{F}_{S_{B}, \text { net }}=\vec{G}_{S_{B}}+\vec{F}_{S_{B}, \text { contact }}=0$. Thus, the force through contact is of the same magnitude as the weight of shaded region $S_{B}$, but with opposite sign $\vec{F}_{S_{B}, \text { contact }}=-\vec{G}_{S_{B}}=-\rho_{1} g V_{S} \hat{z}$. 
The net force exerted on Drop 2 can be obtained by

$$
\begin{aligned}
\vec{F}_{2, \text { net }} & =\vec{G}_{2}+\vec{F}_{2, \text { contact }}=\vec{G}_{2}+\vec{F}_{S_{B}, \text { contact }}, \\
& =\left(\rho_{2} g V_{2}-\rho_{1} g V_{S}\right) \hat{z}
\end{aligned}
$$

where $V_{2}$ is the volume of Drop 2. This equation shows that the net force exerted on Drop 2

contains only a vertical component. There is no closed form expression for an arbitrary

Laplacian shape enclosing the volumes of Drop 2 and the shaded region $S\left(V_{2}\right.$ and $\left.V_{S}\right)$.

An interesting result from Eq. (4) is that a more dense drop resting on top of a less dense is never stable. This can be stated even though there is no closed form expression for the volumes. A net upward force on Drop 2 restores it to the axisymmetric position, producing a stable axisymmetric equilibrium configuration; but Eq. (4) predicts that for a more dense drop resting on top, the net force on Drop 2 once perturbed is always downward for the following reasons. In Figure 3a, because of Neumann's triangle rule, the angle $\theta_{12}$ is always smaller than $\pi$; therefore the volume of Drop 2 is always larger than the volume of the shaded region $S$, i.e. $V_{2}>V_{S}$. The net force on a more dense Drop $2 \vec{F}_{2, \text { net }}=\left(\rho_{2} g V_{2}-\rho_{1} g V_{S}\right) \hat{z}$ is always in the positive $\mathrm{z}$ direction (downward) due to the conditions $\rho_{2}>\rho_{1}$ and $V_{2}>V_{S}$.

This result that a more dense drop cannot rest stably on top of a less dense drop seems to be in contrast with the experimental findings of Phan et $a l^{8,9}$, where more dense drops can float on top of less dense flat fluid interface. In the case of a flat interface, the configuration of a more dense drop floating on top of a flat lower fluid has translational symmetry in the sense that the more dense drop can be stable at any position if it is translated horizontally to any position without changing the total gravitational energy. In contrast, for compound sessile drops, the 
translational symmetry is broken and instead replaced with axisymmetry in the equilibrium configuration. Thus, the apex is intrinsically a unique position at the maximum height, even if a very large bottom drop can appear to have a nearly flat surface near the apex. Therefore, in the case of a compound sessile drop, a more dense drop cannot float stably on top of a less dense drop even though more dense drops are observed to float on top of a less dense flat fluid interface.

\section{Stability criterion of Laplacian shape drops in the zero Bond number limit}

To obtain an analytical expression, calculations are performed in the zero Bond number limit for both Drop 1 and Drop 2. In the zero Bond number limit, where the drop is much smaller than the capillary length, but gravity is still present, the shape is well approximated by a spherical cap to first order accuracy in Bond number ${ }^{24}$.

In the zero Bond limit the volume of Drop 2 is the sum of two spherical caps with basal angles $\delta$ and $2 \pi-\theta_{112}-\theta_{12}-\delta$ as shown in Figure 3a,

$$
V_{2}=q(\delta) R^{3}+q\left(2 \pi-\theta_{112}-\theta_{12}-\delta\right) R^{3},
$$

where $q(\theta)=\left(2-3 \cos (\theta)+\cos ^{3}(\theta)\right) \csc (\theta)^{3} \pi / 3$ is a function representing the volume of a spherical cap with basal angle $\theta$ and unit basal radius; $R$ is the basal radius of the spherical cap, and $\delta$ is the angle between the base of the spherical cap and the interface between Drop 1 and Drop 2 as shown in Figure 3a. The angles $\theta_{12}$ and $\theta_{112}$ are the Neumann's angles introduced in Figure 2, also shown in Figure 3a. In the zero Bond number limit, the angle $\delta$ is determined based on geometric relationships to be a function of the volume ratio and the ratios of interfacial tensions ${ }^{16}$. The volume $V_{S}$ of the shaded region $S$ is calculated similarly as the combination of 
two spherical caps with basal angles $\delta$ and $\pi-\theta_{12}-\delta$ :

$$
V_{s}=q\left(\pi-\theta_{12}-\delta\right) R^{3}+q(\delta) R^{3} .
$$

Thus the net force exerted on the top drop (Eq.(4)) is given by:

$$
\vec{F}_{2, \text { net }}=R^{3}\left\{\rho_{2} g\left[q(\delta)+q\left(2 \pi-\theta_{12}-\theta_{12}-\delta\right)\right]-\rho_{1} g\left[q\left(\pi-\theta_{12}-\delta\right)+q(\delta)\right]\right\} \hat{z},
$$

Setting the net force equal to zero gives a critical condition for the density ratio:

$$
\left.\frac{\rho_{2}}{\rho_{1}}\right|_{\text {critical }}=\frac{q\left(\pi-\theta_{12}-\delta\right)+q(\delta)}{q(\delta)+q\left(2 \pi-\theta_{112}-\theta_{12}-\delta\right)} .
$$

Since angles $\theta_{12}$ and angle $\theta_{112}$ are the Neumann's angles determined by ratios of interfacial tensions and angle $\delta$ depends on the volume ratio and contact angle through geometric relationships ${ }^{16}$, the critical density ratio is then dependent on volume ratio, two interfacial tension ratios, and the contact angle at the solid surface.

A net upward force on Drop 2 restores it to the axisymmetric position, producing an energy minimum or a stable axisymmetric equilibrium configuration. When the density ratio is less than the critical value given in Eq. (6), the axisymmetric configuration is stable. A net downward force on Drop 2 causes it to slide further away from the axisymmetric position, producing an energy maximum or an unstable axisymmetric equilibrium configuration. Thus when the density ratio is greater than the critical density ratio, the axisymmetric configuration is unstable. In the following sections, we will use simulations and experiments to verify the stability conditions.

The derivation of the net force on Drop 2 does not depend on the value of $\beta$, the angle by which Drop 2 is perturbed from the axisymmetric position. Therefore for compound sessile drops of Laplacian shapes in the zero Bond number limit, if the density ratio is not equal to the critical 
value, then there will be no equilibrium configuration other than the axisymmetric configuration. In this case the axisymmetric configuration is a unique extremum which is either a global minimum or a global maximum. At the energy maximum, any finite perturbations will cause Drop 2 to fall away from the apex. At the energy minimum, any finite perturbations that do not cause Drop 2 to touch the solid surface will restore it to the axisymmetric configuration.

The stability criterion (Eq. (6)) in the zero Bond number limit depends on the volume ratio of the two drops $V_{2} / V_{1}$, two interfacial tension ratios $\gamma_{2 A} / \gamma_{1 A}$ and $\gamma_{12} / \gamma_{1 A}$, and the contact angle $\alpha$ implicitly as described above. Figure 4 shows the dependence of the critical density ratio on the interfacial tension ratios with fixed volume ratio $V_{2} / V_{1}=0.2$, and fixed contact angle $\alpha=90^{\circ}$ as an example. Each curve represents the critical density ratio as a function of the ratio $\left(\gamma_{12}-\gamma_{1 A}\right) / \gamma_{2 A}$ for fixed ratio $\gamma_{2 A} / \gamma_{1 A}$. Since the three phase contact line between Drop 1, Drop 2 and the ambient phase must obey the Neumann condition, the allowed range of $\gamma_{12} / \gamma_{1 A}$ values is different for each $\gamma_{2 A} / \gamma_{1 A}$ value, but the allowed values of $\left(\gamma_{12}-\gamma_{1 A}\right) / \gamma_{2 A}$ lie between $(-1,1)$ for any given surface tension ratio $\gamma_{2 A} / \gamma_{1 A}$. For a fixed surface tension ratio $\gamma_{2 A} / \gamma_{1 A}$, the critical density ratio is a monotonically decreasing function of the ratio of interfacial tensions $\left(\gamma_{12}-\gamma_{1 A}\right) / \gamma_{2 A}$. For fixed surface tensions, this parameter effectively represents the interfacial tension between Drop 1 and Drop 2. The critical density ratio is close to unity for a surface tension ratio of unity and the interfacial tension ratio near $\left(\gamma_{12}-\gamma_{1 A}\right) / \gamma_{2 A} \approx-1$. For a fixed value of $\left(\gamma_{12}-\gamma_{1 A}\right) / \gamma_{2 A}$, the critical density ratio decreases with increasing surface tension ratio $\gamma_{2 A} / \gamma_{1 A}$. The inset of Figure 4 shows the rescaled critical 


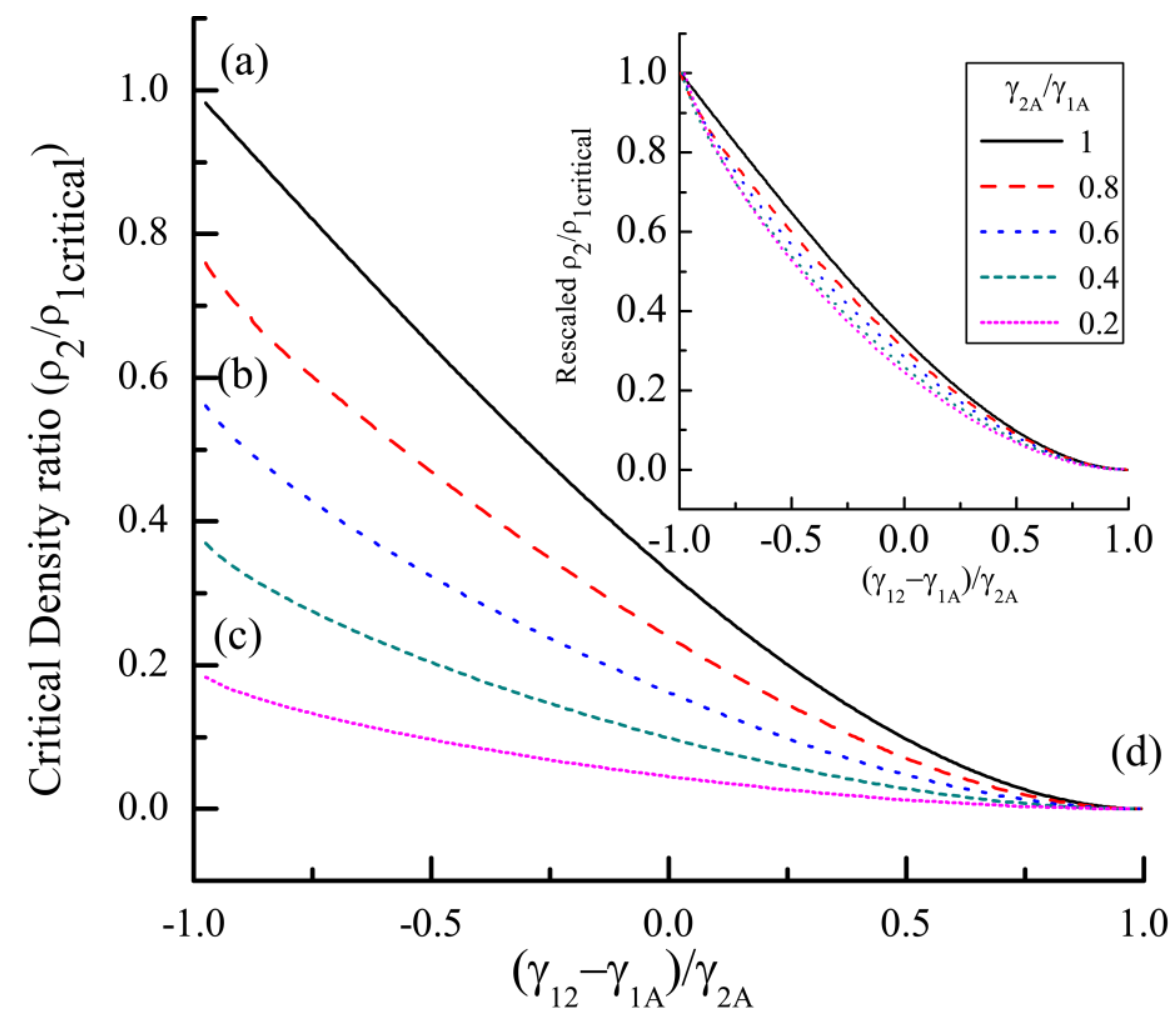

(a)

(b)

(c)

(d)
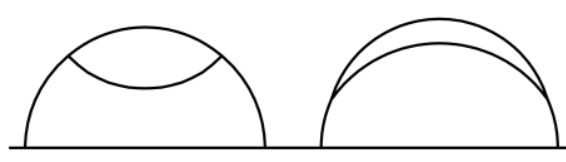

Figure 4. Critical density ratio as a function of surface and interfacial tensions with fixed volume ratio $V_{2} / V_{1}=0.2$, and fixed contact angle $\alpha=90^{\circ}$. Legend denotes the ratio of the top surface tension divided by the bottom surface tension. (a),(b), and (c) indicate the configurations of spherical cap shapes with Neumann's angles calculated for the corresponding interfacial tensions. ( (a) $\gamma_{2 A} / \gamma_{1 A}=1$, (b) $\gamma_{2 A} / \gamma_{1 A}=0.6$, (c) $\gamma_{2 A} / \gamma_{1 A}=0.2$. For (a), (b) and (c), the value $\left(\gamma_{12}-\gamma_{1 A}\right) / \gamma_{2 A}=-0.98$ is kept constant. The configuration for (d) is obtained for dewetting drops with $\gamma_{2 A} / \gamma_{1 A}=1$ and $\left(\gamma_{12}-\gamma_{1 A}\right) / \gamma_{2 A}=0.98$.

density ratio based on the linear function, rescaledratio $=\left(\right.$ ratio - ratio $\left._{\min }\right) /\left(\right.$ ratio $_{\max }-$ ratio $\left._{\text {min }}\right)$, where ratio $_{\text {min }}$ and ratio $_{\text {max }}$ represent the minimum and maximum critical density ratios within the allowed range of $\left(\gamma_{12}-\gamma_{1 A}\right) / \gamma_{2 A}$ values. The rescaled critical density ratio lies between 0 and 1 . The systematic deviation of the rescaled ratio suggests that the critical density ratio is not 
a simple function of the two ratios, $\left(\gamma_{12}-\gamma_{1 A}\right) / \gamma_{2 A}$ and $\gamma_{2 A} / \gamma_{1 A}$, but rather that both are important in determining the critical conditions for compound drop stability.

In Figure 4, (a), (b), (c) and (d) denote the configurations of compound sessile drops with spherical cap shapes and Neumann's angle conditions resulting from the specified interfacial tension ratios. The surface tension ratios for configurations (a), (b) and (c) are $\gamma_{2 A} / \gamma_{1 A}=1,0.6$, and 0.2 respectively while the interfacial tension ratio is fixed at $\left(\gamma_{12}-\gamma_{1 A}\right) / \gamma_{2 A}=-0.98$, indicating nearly the smallest interfacial tension achievable while still obeying the Neumann's triangle condition. Configuration (d) represents dewetting drops with very large interfacial tension $\left(\gamma_{12}-\gamma_{1 A}\right) / \gamma_{2 A}=0.98$, and surface tension ratio $\gamma_{2 A} / \gamma_{1 A}=1$. However even for other values of $\gamma_{2 A} / \gamma_{1 A}$, the shapes obtained remain quite similar to that shown in (d) because the angle between the two surfaces is close to zero, while the angles between the surfaces and the interface are both close to $180^{\circ}$. These configurations agree with the physical intuition that dewetting drops are nominally less stable. This intuition is supported by the calculation that the critical density ratio is close to zero in this limit. For drops with small interfacial tensions, near $\left(\gamma_{12}-\gamma_{1}\right) / \gamma_{2}=-0.98$, the drop shapes favor more contact of the two phases, therefore promoting greater stability.

Figure 4 suggests that the critical density ratio is larger for low interfacial tension systems and smaller for high interfacial tension systems. From the point of view of real systems, combinations of organic liquids (oil) and inorganic liquids (water) typically exhibit large interfacial tensions, while separated phases obtained by mixing partially miscible fluids exhibit relatively small interfacial tension values ${ }^{34}$. In the following sections we will consider both small 
and large interfacial tension systems separately in both simulation and experiments. While the derivation of the stability criterion is based on drops of Laplacian shape in the zero Bond number limit, we will see in the simulation and experimental results that the stability criterion for the limiting case provides a reasonable estimate even when the drop shapes are not constrained to Laplacian shapes, and even when the Bond number increases.

\section{Simulation Results Using Surface Evolver}

The zero Bond number analysis shows that the critical density ratio separating the energy maxima from the energy minima depends on the interfacial tension: larger interfacial tension yields a smaller critical density ratio. To verify the stability criterion, we conduct numerical simulations for both high and low interfacial tension systems. Because real experimental systems typically have fluid and interfacial properties that cannot be varied independently, simulations allow the independent roles of these properties to be isolated. Further, simulations can be used to examine finite Bond number situations.

The Laplace equation can be derived using a variational method based on the minimization

of Gibbs free energy ${ }^{4,25,35}$. Thus the configuration of a compound sessile drop satisfying the three Laplace differential equations can be solved numerically in this framework by minimizing the free energy. We utilize the open access software package Surface Evolver developed by Brakke $^{18,19}$, which models the liquid surface shapes subject to various forces or constraints. We developed code within the software adapted to the compound sessile drop geometry. Unlike the zero Bond number stability analysis in which we assumed the drops have Laplacian shape, in Surface Evolver we examine drop shapes that are perturbed to start with spherical cap shapes. 
(a)

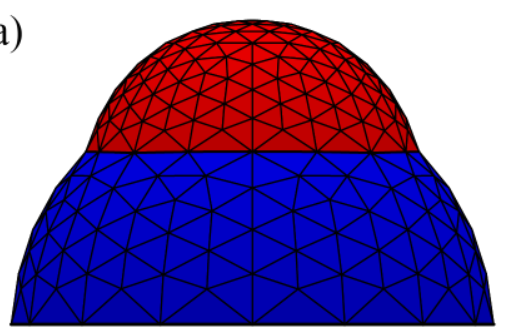

(c)

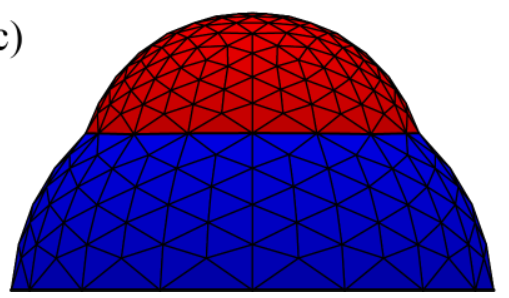

(b)

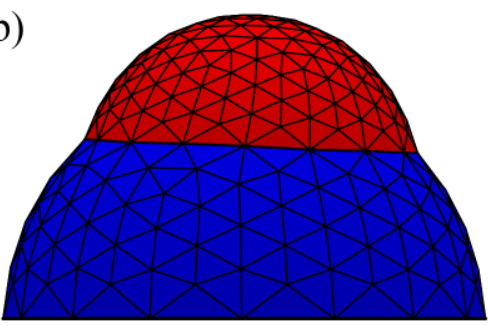

(d)

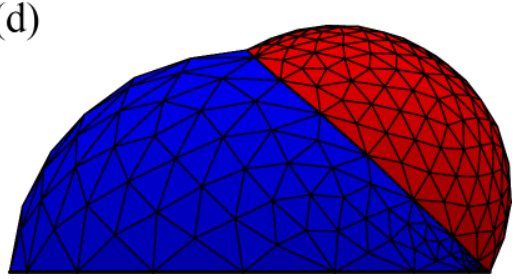

Figure 5. Protocol for determining whether the axisymmetric state is an energy maximum or minimum using Surface Evolver. (a) Initial axisymmetric equilibrium shape without gravity. (b) Top drop displaced from the vertical axis by about 3 degrees without gravity. (c) Upon application of gravity at small density ratios, the top drop returns to the apex position, indicating a minimum energy system. (d) Upon application of gravity at large density ratios, the top drop slides off, indicating a maximum energy state.

This slightly different type of perturbation can provide a complementary perspective as to the influence of different perturbations on the stability.

The procedure for examining the stability of the axisymmetric configuration of the compound sessile drop is outlined in Figure 5. The equilibrium axisymmetric configuration is first obtained in a zero gravity environment, and then the top drop is displaced away from the axisymmetric position. Lastly, gravity is applied, and the evolution of the top drop is monitored. If the top drop slides off it is recorded as an unstable configuration, and if it restores to the apex it is considered stable. This perturbation bears some similarity to experiments in which the top drop is perturbed using a pipette tip, where gravity is always present. However, in simulation, the perturbed drop shapes are constrained to spherical caps, while in experiments the perturbed drop shape is affected by the wetting properties of the pipette. The convergence of simulations is 
supported by the nearly constant energy output and scale after multiple runs as well as mesh refinement. As Surface Evolver uses a gradient descent method ${ }^{18}$ to search for the energy minimum, it may occur that the final configuration is a local minimum instead of the global minimum. However, the identification of a local minimum is sufficient to specify the stability at the axisymmetric configuration. Based on the gradient search direction, evolution away from the apex position indicates that the axisymmetric configuration is unstable while evolution toward the apex indicates that the axisymmetric configuration is stable.

In Figure 5, the surface tension of the smaller drop is $\gamma_{2 A}=25 \mathrm{mN} / \mathrm{m}$, the surface tension of the larger drop is $\gamma_{1 A}=72 \mathrm{mN} / \mathrm{m}$, and the interfacial tension is $\gamma_{12}=52 \mathrm{mN} / \mathrm{m}$. These surface and interfacial tension values correspond to a dodecane drop resting on a water drop in air; however, in simulations the fluid densities are varied. The dimensionless surface tension ratios for this case are $\gamma_{2 A} / \gamma_{1 A}=0.35$ and $\left(\gamma_{12}-\gamma_{1 A}\right) / \gamma_{2 A}=-0.8$. The contact angle for the larger drop against the solid substrate is fixed at $\alpha=90^{\circ}$. The volumes of the two drops are $1 \mu \mathrm{L}$ and 5 $\mu \mathrm{L}$, respectively. Figure 5a shows the equilibrium shape of the compound sessile drop obtained in zero gravity. In the absence of gravity, the shapes obtained from Surface Evolver are similar to the compound drop cases with no solid substrate examined by Guzowski et $a l^{36}$, which are found to be independent of the fluid densities. To examine the stability of the equilibrium axisymmetric position, the top drop is displaced about $3^{\circ}$ from the vertical axis and allowed to equilibrate without gravity as shown in Figure 5b. Gravity is then applied to this perturbed state to examine the stability. By varying the densities of the two drops, we expect that the application of gravity will cause the interface to evolve in one of two ways: either it will restore to the axisymmetric 
configuration (Figure 5c), or it will slide off to the side, away from the apex (Figure 5d). Note that the configuration shown in Figure 5d does not represent the equilibrium state. In the current setup, Surface Evolver does not allow for a change of topology to accommodate the contact of the smaller drop with the solid surface. In real systems, when the deposited top drop contacts the solid surface, a "Janus" configuration is formed, e.g., for the combination of perfluooctane and mercury $^{12}$. The equilibrium configuration of a Janus type compound sessile drop has been modeled using a phase field modeling approach, which is capable of depicting the different wetting properties of the fluids on the solid surface ${ }^{15}$. As we are presently interested in the stability of the axisymmetric configuration, the movement of the second drop away from the axisymmetric position is sufficient to demonstrate the lack of stability of the configuration.

As shown in Figures $5 \mathrm{c}$ and $5 \mathrm{~d}$, depending on the densities at fixed surface tensions, interfacial tension, contact angle and volumes, the axisymmetric equilibrium can be categorized as either an energy minimum or an energy maximum. Note that the drop shapes shown in Figure $5 \mathrm{c}$ are flattened compared with those of Figure 5a owing to the presence of gravity. Using several examples, we confirmed that the shape of the axisymmetric configuration at equilibrium is the same if the seven governing dimensionless parameters are kept fixed while varying the dimensional values of the physical properties. Note that when the density ratio used in simulation is close to the critical density ratio, the convergence rate for evolution of the interface is very slow. Thus to further narrow in on the dividing line, for density ratios close to the critical value, 
(a)

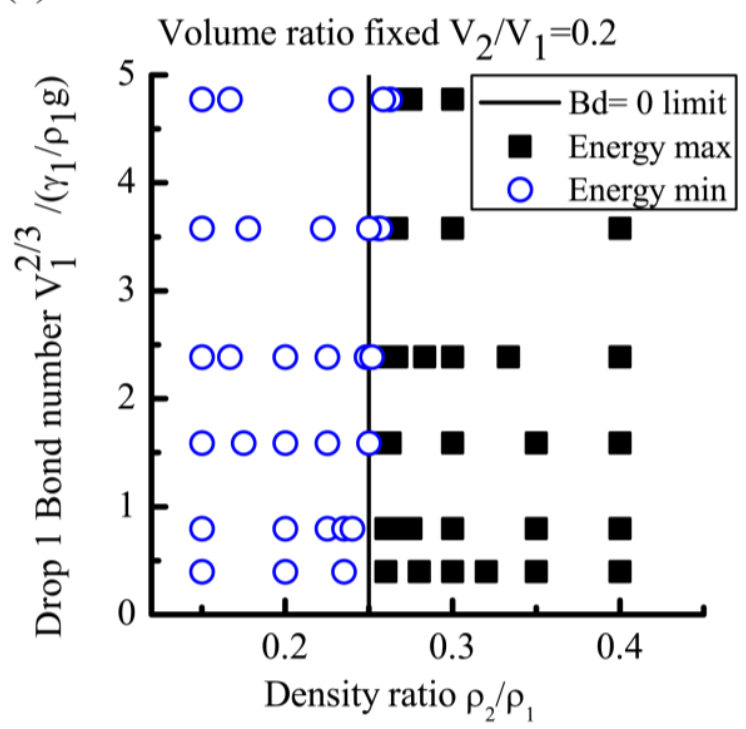

(b)

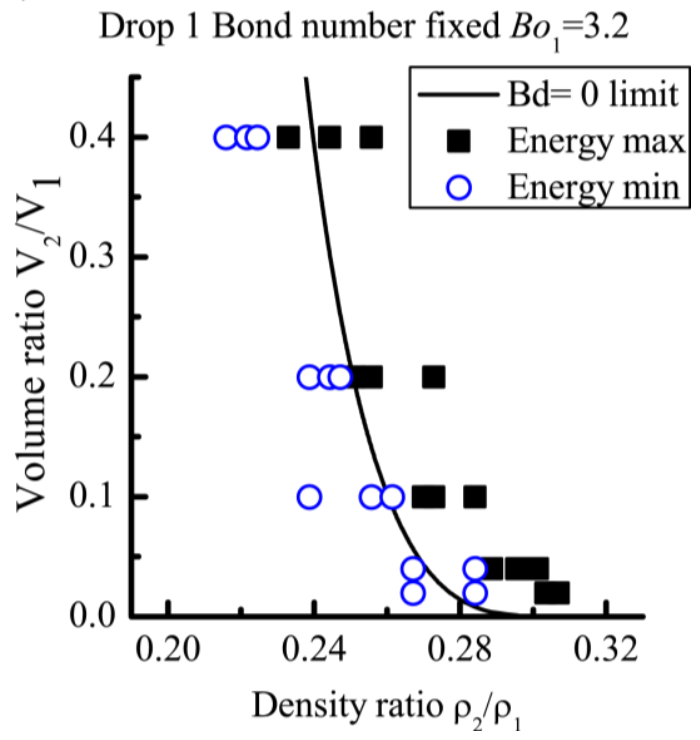

Figure 6. The energy maximum and minimum states obtained from Surface Evolver for a high interfacial tension system $\left(\gamma_{1 A}=72 \mathrm{mN} / \mathrm{m}, \gamma_{2 A}=25 \mathrm{mN} / \mathrm{m}, \gamma_{12}=52 \mathrm{mN} / \mathrm{m}\right)$. (a) The volume of the top drop is $1 \mu \mathrm{L}$ and the bottom drop is $5 \mu \mathrm{L}$. The densities of the two drops are varied to achieve different density ratios and Bond numbers. The critical density ratio deviates from the zero Bond number asymptotic prediction as the Bond number increases. (b) The Bond number of the bottom drop is fixed at an intermediate value of 3.2 and the transition from an energy minimum to an energy maximum deviates slightly from the zero-Bond number model at various volume ratios.

unstable cases have been repeated with a smaller perturbation of $1^{\circ}$ from the apex. Only those configurations that remain unstable at a $1^{\circ}$ displacement are marked as energy maxima.

Figure 6a summarizes the simulation results for the high interfacial tension system considered in Figure 5. The densities of the two drops are varied to achieve different ratios as well as different Bond numbers. In Figure $6 a$, the stability of each configuration is noted as a function of the density ratio and the Bond number of Drop 1, for a fixed volume ratio of 0.2. Stable configurations are marked by open symbols, while unstable configurations are marked by filled symbols. The simulations result in stable configurations at lower density ratios and unstable configurations at higher density ratios, with a clear transition between the two regions. 
The vertical line indicates the predicted critical density ratio for this system at fixed volume ratio of 0.2 using the zero Bond number calculation. The figure shows that the zero Bond number approximation predicts the transition from an energy minimum to an energy maximum reasonably well at small Bond number, while a small deviation is observed at increasing Bond number. At a Bond number of $B o_{1} \approx 5$ for Drop 1, the deviation from the zero Bond number predictions is about $4 \%$. This small deviation results from the gravity-driven flattening of the bottom drop, which tends to stabilize the axisymmetric configuration up to a slightly larger critical density ratio.

Based on the earlier dimensional analysis, the critical density ratio also depends on the volume ratio. Figure $6 \mathrm{~b}$ examines the stability of the axisymmetric configuration for a fixed Bond number of Drop 1 of $B o_{1}=3.2$, plotting the stability of individual configurations on a plot of volume ratio versus density ratio. As in Figure 6a, the stable configurations are found at lower density ratios, while unstable configurations are found at higher density ratios, with a clear transition between the two regions. In this case, the stability transition depends on volume ratio, shifting to lower critical density ratio values for larger volume ratios. The solid line represents the critical density ratio predicted in the zero Bond number limit. While the same trend is observed, the simulations exhibit a small deviation from this limit. The deviation arises from the slight gravity-induced flattening of both the top and bottom drops at non-zero Bond numbers. The deviation observed in Figure $6 \mathrm{~b}$ at zero Bond number is about $2 \%$ of the critical density ratio. 
(a)

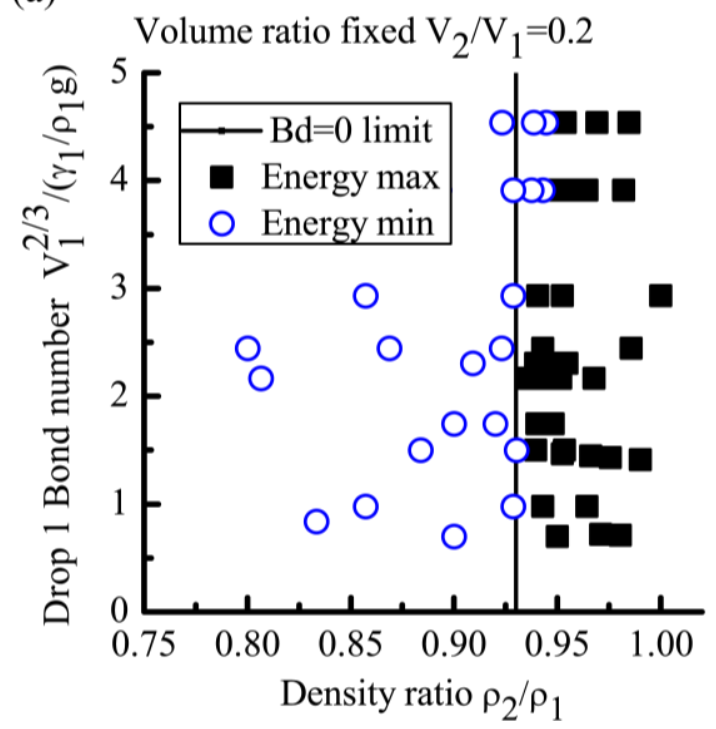

(b)

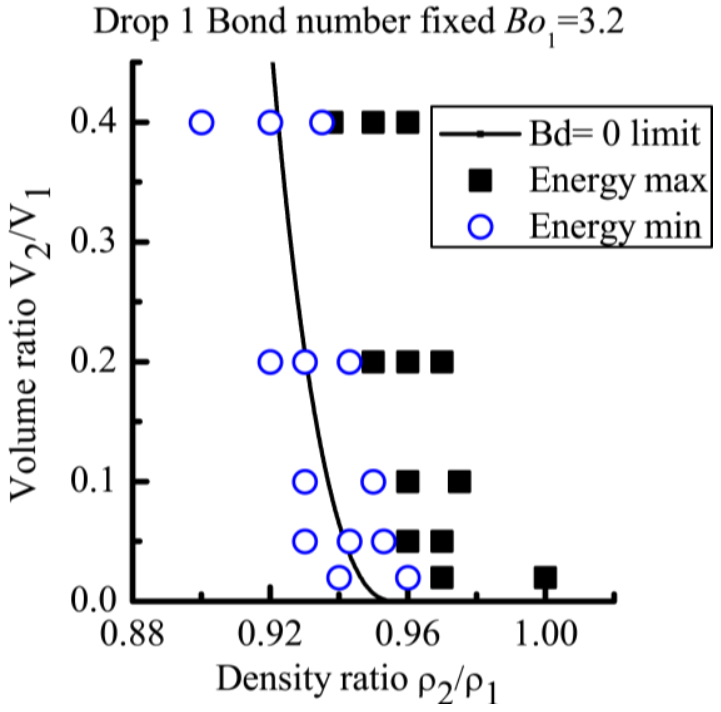

Figure 7. The energy maximum and minimum states obtained from Surface Evolver for a low interfacial tension system $\left(\gamma_{1 A}=41 \mathrm{mN} / \mathrm{m}, \gamma_{2 A}=40 \mathrm{mN} / \mathrm{m}, \gamma_{12}=3 \mathrm{mN} / \mathrm{m}\right)$. (a) The volume ratio of the top drop to the bottom drop is fixed at 0.2 , and the densities of the two drops are varied to achieve different Bond numbers, yielding different maximum and minimum energy states. The critical density ratio deviates from the zero Bond number asymptotic prediction as the Bond number increases. (b) The Bond number of the bottom drop is fixed at an intermediate value of 3.2 and the transition from an energy minimum to an energy maximum deviates from the zero-Bond number limit at all volume ratios.

Figure 7 summarizes the simulation results for a low interfacial tension system. In this case, the surface tension is $\gamma_{2 A}=40 \mathrm{mN} / \mathrm{m}$ for the smaller drop and $\gamma_{1 A}=41 \mathrm{mN} / \mathrm{m}$ for the larger drop. The interfacial tension is $\gamma_{12}=3 \mathrm{mN} / \mathrm{m}$. The dimensionless surface tension ratios for this case are $\gamma_{2 A} / \gamma_{1 A}=0.98$ and $\left(\gamma_{12}-\gamma_{1 A}\right) / \gamma_{2 A}=-0.95$. The contact angle for the larger drop against the solid substrate is fixed at $\alpha=90^{\circ}$. In Figure $7 \mathrm{a}$, the volume ratio is held fixed at a value of 0.2 , and the stable and unstable configurations are marked with open and filled symbols, respectively, as a function of the Bond number of Drop 1 and the density ratio. The simulation results demonstrate the same features as for the high interfacial tension case: i.e., the stable configurations are organized at lower density ratios, while the unstable configurations are 
organized at higher density ratios, with a clear transition between the two regions. The zero Bond number approximation, denoted by the vertical line, agrees well with the critical density ratio observed in simulations. The critical value found in simulations deviates about $2 \%$ from the zero Bond number value at $B o_{1} \approx 5$ for Drop 1. In Figure $7 \mathrm{~b}$, the Bond number of Drop 1 is held fixed at $B o_{1}=3.2$, while the volume ratio varies. In this case, the critical density ratio resulting from the simulations is always larger than the critical density ratio predicted at zero Bond number. This is in contrast to the results shown in Figure 6b, where the critical density ratio from simulation is larger than the zero Bond number value at smaller volume ratios, and smaller than the zero Bond number value at large volume ratios.

The deviation of the critical density ratio in simulations compared with the zero Bond number limit can be attributed to the finite Bond number of Drop 2, $B \mathrm{O}_{2}=V_{2}^{2 / 3} /\left(\gamma_{2} / \Delta \rho_{2} g\right)$. In Figure $6 b$, the ratio of the Bond numbers near the transition line is $B o_{2} / B o_{1}=0.72\left(V_{2} / V_{1}\right)^{2 / 3}$, while the ratio of Bond numbers in Figure $7 \mathrm{~b}$ is $\mathrm{BO}_{2} / \mathrm{BO}_{1}=0.95\left(V_{2} / V_{1}\right)^{2 / 3}$. Thus, even though the volume ratios for the high and low interfacial tension cases are set to the same value, the Bond number ratios are different as a result of the surface and interfacial tension differences. Nevertheless, the predicted critical density ratio at zero Bond number and the critical density ratio resulting from simulation are only $2-4 \%$ different, suggesting that the zero Bond number approximation captures the major factors controlling the transition from an energy maximum to an energy minimum.

The simulations show that the critical density ratio is close to the zero Bond number limit for both high and low interfacial tension systems, with merely $2-4 \%$ deviation for Bond number 
$B o_{1} \approx 5$. For the high interfacial tension system to reach a stable axisymmetric configuration, the density ratio of the two drops must be smaller than 0.25 . For the low interfacial tension system to reach a stable axisymmetric configuration, the density ratio must be smaller than 0.93 . In the following section we conduct experiments for real systems in which we tune the density ratios and realize both stable and unstable axisymmetric configurations.

\section{Experimental Methods and Materials}

The previous analysis and simulations demonstrate that the stability of a compound sessile drop depends strongly on the magnitudes of the surface and interfacial tensions. Validating the predicted critical density ratio in real systems is challenging since fluid and interfacial properties typically cannot be varied independently. In the case of compound metal drops, the density ratio has been adjusted by varying temperature ${ }^{10}$. In aqueous systems, cesium chloride salt can be added to vary the density with minimal viscosity and surface tension changes ${ }^{37,38}$. In this paper, we examine two liquid pairs containing at least one aqueous component, one with high interfacial tension and one with low interfacial tension. In each case the density is varied using different concentrations of cesium chloride salt added to the aqueous phase, allowing the critical density ratio to be determined for a fixed set of surface and interfacial tension values.

The high interfacial tension system consists of dodecane paired with aqueous cesium chloride ( $\mathrm{CsCl}$, purchased from Sigma-Aldrich with purity $\geq 99 \%$, used as received) solutions. Dodecane and water systems have previously been used to verify the Neumann's angle calculation $^{39}$, and exhibit relatively large interfacial tension values of about $52 \mathrm{mN} / \mathrm{m}$. The fluid and interfacial properties of each liquid considered are listed in Table I. Dodecane 
(Sigma-Aldrich, purity $\geq 99 \%$ ) is further purified using column chromatography over aluminum oxide and silica. The chromatography materials are obtained from Sigma-Aldrich and used as received. The $\mathrm{CsCl}$ solutions are prepared by mixing deionized water (Milli-Q, 18M $\Omega-\mathrm{cm}$, organic content $<10 \mathrm{ppb}$ ) with an appropriate mass of $\mathrm{CsCl}$. Densities are determined by measuring the mass (Denver Instrumental Company Balance, Model XE-100A, accuracy $10^{-4} \mathrm{~g}$ ) of a known volume contained in a volumetric flask (Pyrex No. 5640, $25 \pm 0.03 \mathrm{ml}$ ). Experimental uncertainty is estimated based on propagation of error from different experimental sources, including instrumental error from the volumetric flask of about $0.1 \%$ with statistical error is about $0.05 \%$, and instrumental error from the mass balance of about $0.02 \%$ with statistical error about $0.03 \%$. Estimated uncertainties are in the fourth decimal place of the reported density values. Surface and interfacial tension values are measured using a Du Noüy ring tensiometer ${ }^{40}$. The measured values for dodecane and water agree well with reported literature values ${ }^{39}$.

The low interfacial tension system is obtained by inducing phase separation of partially miscible fluids ${ }^{34,41}$. Low interfacial tension values are common in colloid-polymer ${ }^{42,43}$ and polymer-polymer ${ }^{44,45}$ mixtures that undergo phase separation, leading to two immiscible liquid components with interfacial tension values as low as $1 \mu \mathrm{N} / \mathrm{m}$. Mixing water with alcohols such as benzyl alcohol or 1-butanol leads to phase separation into water-rich and alcohol-rich liquid phases with interfacial tension values of $1-4 \mathrm{mN} / \mathrm{m} \cdot{ }^{34,41,46}$ Although the phase diagram of the 1-butanol-water mixture is well characterized ${ }^{46}$ and the interfacial tension value is only 1.8 $\mathrm{mN} / \mathrm{m}^{34}, 1$-butanol has a relative high vapor pressure of $665 \mathrm{~Pa}$ at $20^{\circ} \mathrm{C}{ }^{47}$. To avoid evaporation 
in the compound sessile drops, we select benzyl alcohol, which exhibits a low vapor pressure of $12 \mathrm{~Pa}$ at $25^{\circ} \mathrm{C}^{48}$. The benzyl alcohol is obtained from Sigma-Aldrich (purity $\geq 99 \%$ ) and used as received. To form the immiscible liquid pairs, aqueous $\mathrm{CsCl}$ solutions are mixed with benzyl alcohol at similar volumes. Upon equilibration, the mixture separates into two phases, one that is water and salt rich, and one that is water and salt rare. The two phases are collected using a separation funnel. The water rich and water rare phases are identified by miscibility: the water rich phase is miscible with addition of water, but separates into two phases if extra benzyl alcohol is added.

Table II lists the densities measured for each of the two liquid phases obtained by mixing different concentrations of $\mathrm{CsCl}$ solution with benzyl alcohol. The density is measured using the method described above. Note that the water rich phase obtained from the benzyl alcohol-water mixture is of lower density than the water rare phase, because benzyl alcohol is denser than water. However, since $\mathrm{CsCl}$ salt is more soluble in the water rich phase, the water rich phase obtained from the benzyl alcohol-CsCl solution is denser than the water rare phase without $\mathrm{CsCl}$, as seen in the second and third row of Table II. The density ratio of the less dense fluid to that of the more dense fluid first increases from 0.964 to approximately 1 , and then decreases to about 0.826 as the concentration of $\mathrm{CsCl}$ increases. The highest concentration of $\mathrm{CsCl}$ obtained in solution is $26 \%$. For concentrations greater than $30 \%$, we observe crystallization of $\mathrm{CsCl}$ upon mixing with benzyl alcohol, even though in principle saturation is not achieved until $60 \% \mathrm{CsCl}$ in the two-element $\mathrm{CsCl}$-water system ${ }^{37}$. The measurements of surface and interfacial tensions are listed in Table III, where the water rich phase always exhibits a higher surface tension than the 
water rare phase.

Compound sessile drops are examined for the fluid pairs discussed, and are placed on solid hydrophobic surfaces exhibiting a contact angle of $102 \pm 5^{\circ}$ with deionized water. The surfaces are prepared by applying Aquapel ${ }^{\mathrm{TM}}$ (PPG Industries) to microscope glass slides (Fisherbrand 12-567) following the manufacturer instructions. The surface is robust against rinsing with ethanol or acetone.

The formation and stability of compound sessile drops are recorded using a CCD camera (Q-See, Anaheim CA, model no. QPSCDNV, 30 fps mounted with a 55mm telecentric lens from Edmund Optics) oriented at a $45^{\circ}$ angle to the horizontal surface. A first drop of specified volume $(10-100 \mu \mathrm{L})$ is deposited on the hydrophobic surface using a pipette (Eppendorf $100 \mu \mathrm{L}$ pipette with maximum systematic and random error of $0.8 \pm 0.3 \mu \mathrm{L}$ ) and allowed to reach its equilibrium configuration. A second drop of smaller volume $(2-80 \mu \mathrm{L})$ is deposited using two different pipettes depending on the desired volume (Eppendorf $20 \mu \mathrm{L}$ pipette with error $0.2 \pm 0.1$ $\mu \mathrm{L}$, or Eppendorf $100 \mu \mathrm{L}$ pipette with error $0.8 \pm 0.3 \mu \mathrm{L}$ ). Upon deposition, if the second drop slides off the apex of the lower drop, then this combination is documented as an unstable configuration. If the second drop remains at the axisymmetric configuration, then the pipette tip is used to further perturb the top drop from the apex without permitting it to touch the solid substrate. If the second drop restores to the apex at least three times, then this combination is documented as a stable axisymmetric configuration. If the restoration cannot be repeated, the combination is documented as an uncertain condition. The Bond number of the bottom drop is in the range of $1<B o_{1}<7$. 


\section{Experimental Results and Discussion}

Both the zero Bond number analysis and Surface Evolver simulations demonstrate that the stability of an equilibrium axisymmetric configuration can be predicted by comparing the density ratio of two drops with the critical density ratio calculated for the specified fluid system. The critical density ratio of a high interfacial tension system is significantly different from that of a low interfacial tension system. In this section, we first describe the experimental realization of stable and unstable compound sessile drops for both the low and high interfacial tension systems described in the previous section. The transition from stable to unstable configurations is then compared quantitatively with the asymptotic approximation and the simulations.

For the high interfacial tension system consisting of dodecane and aqueous $\mathrm{CsCl}$ solutions, all the experiments exhibit unstable axisymmetric configurations: once the smaller drop is deposited on top of the larger drop, it slides off to the side and forms a Janus configuration. A typical experiment is shown in Video 3 of the Supplementary Materials. From the zero Bond number analysis, the critical density ratio is approximately $0.24-0.30$ for all of the volume ratios considered. However, experimentally, the accessible density ratio range is $0.41-0.75$, well above the theoretical transition value. Thus, the observation that no stable axisymmetric configurations are experimentally realized for the high interfacial tension system is in agreement with both analysis and simulations.

For the low interfacial tension system of the phase separated mixtures of benzyl alcohol and aqueous $\mathrm{CsCl}$ solutions, both unstable and stable axisymmetric configurations are observed. As

shown in Figure 1a, for the liquid pairs obtained by mixing benzyl alcohol and 22\% aqueous 
(a)

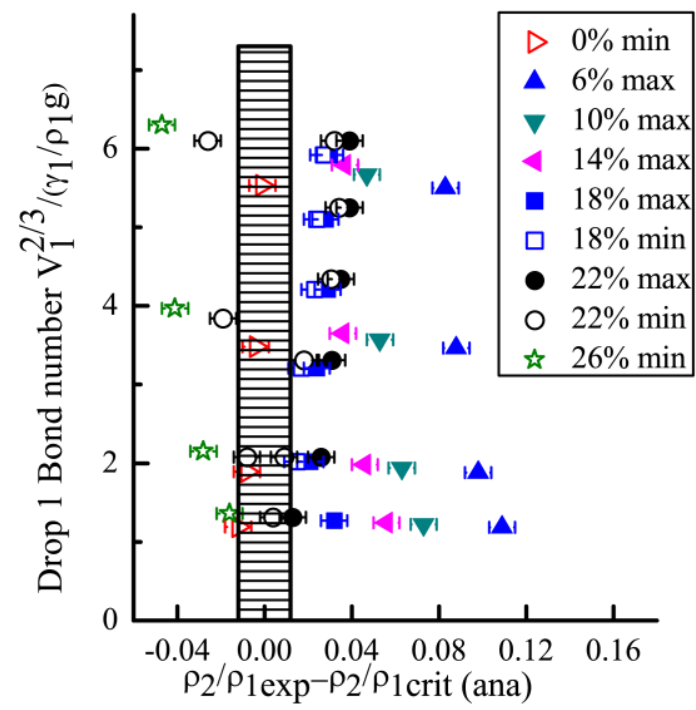

(b)

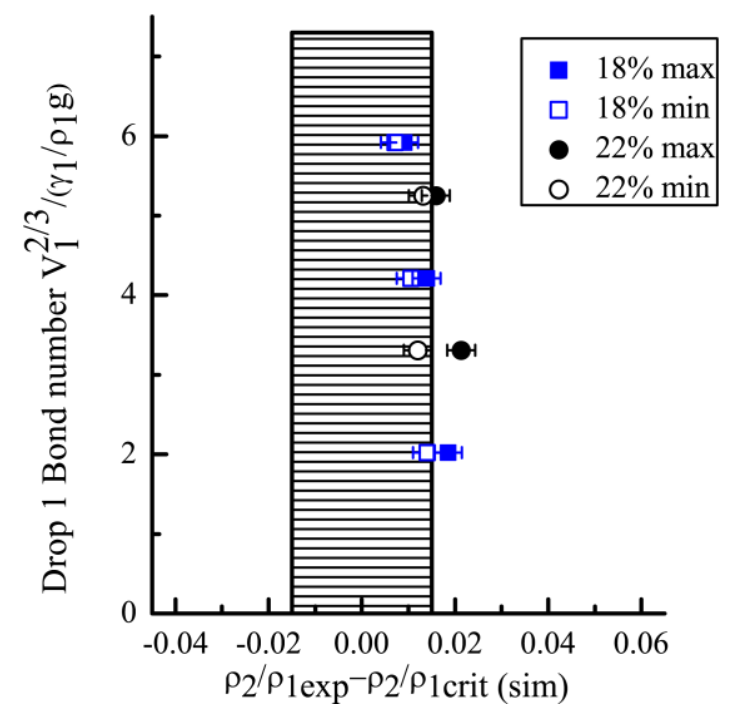

Figure 8. a) Summary plot of benzyl alcohol experiments compared with the zero Bond number analytical model. The vertical axis is the Bond number of Drop 1 and the horizontal axis represents the difference between the experimental density ratio and the critical density ratio predicted in the zero Bond number limit. The open symbols denote energy minima while the filled symbols denote energy maxima. b) Comparison of experimental results with Surface Evolver simulations for the systems using $18 \%$ and $22 \% \mathrm{CsCl}$ solutions. The horizontal axis indicates the difference between the experimental density ratio and the critical density ratio obtained in simulations.

$\mathrm{CsCl}$ solution, the experimental density ratio is 0.859 , less than the predicted critical density ratio of $\rho_{2} / \rho_{\text {lcrit(ana) }}=0.878$. Deposition of a drop of the water rare phase onto a drop of the water rich phase results in an axisymmetric configuration that is stable against perturbations from the apex. Video 1 of the Supplementary Materials shows a typical example of a stable configuration. In contrast, as shown in Figure 1b, for the liquid pairs obtained by mixing benzyl alcohol and $10 \%$ aqueous $\mathrm{CsCl}$ solution, the experimental density ratio is 0.955 , greater than the predicted critical density ratio of $\rho_{2} / \rho_{\text {lcrit(ana) }}=0.896$. Deposition of a drop of the water rare phase onto a drop of the water rich phase yields an unstable axisymmetric configuration as shown in Video 2 of the Supplementary Materials). The observed transition from an energy minimum to an energy 
maximum allows for quantitative experimental comparisons with both the analysis and the simulations.

Figure 8 summarizes the experiments including both stable and unstable axisymmetric configurations for the phase separated mixture of benzyl alcohol and varying concentrations of aqueous $\mathrm{CsCl}$ solution. Each symbol denotes a liquid pair obtained from the phase separated mixture with the specified concentration of $\mathrm{CsCl}$. The open symbols represent stable axisymmetric configurations (energy minimum) and the filled symbols represent unstable axisymmetric configurations (energy maximum). The volumes of both drops are varied to achieve different Bond numbers of the bottom drop $B o_{1}$ and different volume ratios. The Bond number of Drop 1 has a practical lower limit $B o_{1} \approx 1$, because smaller drops are difficult to deposit using the pipettes described earlier. All of the experiments consider the deposition of a less dense fluid drop onto a more dense fluid drop because the opposite case, deposition of a more dense drop onto a less dense drop, is always unstable as predicted by Eq. (6).

Figure 8a compares the experimental results with the zero Bond number analysis. The critical density ratios in the zero Bond number limit ((Eq. (6)) are calculated using the relevant experimental values of surface and interfacial tensions, volume ratios and contact angle for each fluid pair. In the experiments, the experimental density ratio is determined once the fluid pair is selected; in contrast, the analysis formulates the critical density ratio as a function of the volume ratio. The horizontal axis of Figure $8 \mathrm{a}$ represents the density ratio difference $\rho_{2} / \rho_{\text {lexp }}-\rho_{2} / \rho_{\text {lcrit(ana) }}$ subtracting the predicted critical density ratio from the experimental 
density ratio. The vertical axis represents the Bond number of Drop $1 B q_{1}=V_{1}^{2 / 3} /\left(\gamma_{1 A} / \triangle q_{A} g\right)$. For all of the drop volumes tested, the axisymmetric configurations for the liquid pairs obtained by mixing benzyl alcohol with $0 \%$ and $26 \%$ aqueous $\mathrm{CsCl}$ solutions are always stable. The axisymmetric configurations for the liquid pairs obtained by mixing benzyl alcohol with $6 \%, 10 \%$ and $14 \%$ aqueous $\mathrm{CsCl}$ solutions are always unstable. Transitions from an energy minimum to an energy maximum are observed for the liquid pairs obtained using $18 \%$ and $22 \%$ aqueous $\mathrm{CsCl}$ solutions. From the zero Bond number analysis, a positive value of the density ratio difference $\rho_{2} / \rho_{\text {lexp }}-\rho_{2} / \rho_{\text {lcrit(ana) }}$ is expected to result in an energy maximum, while a negative value is expected to result in an energy minimum. The uncertainty in the experimental density ratio is about \pm 0.003 due to the uncertainty in the density measurements. The vertical shaded area shown in Figure 8a near zero on the horizontal axis indicates the expected stable-to-unstable transition, reflecting the estimated uncertainty $(\approx \pm 0.012)$ in calculating $\rho_{2} / \rho_{\text {1crit(ana) }}$ based on the uncertainty in the surface and interfacial tensions and in the volume ratios used in Eq. (6). For the cases in which the Bond number is near unity, $B o_{1} \approx 1$, the transition from an energy minimum to an energy maximum occurs at $\rho_{2} / \rho_{\text {exp }}-\rho_{2} / \rho_{\text {lait(ana) }} \approx 0$, suggesting that the experimental results agree well with the zero Bond number analysis. For the larger Bond number cases, $B o_{1} \approx 6$, the experimental transition occurs at $\rho_{2} / \rho_{\operatorname{lexp}}-\rho_{2} / \rho_{\text {1crit(ana) }} \approx 0.03$, so the experiments deviate slightly from the zero Bond number prediction.

The experimental results are also compared with finite Bond number Surface Evolver simulations in Figure $8 \mathrm{~b}$ for the fluid systems obtained by mixing $18 \%$ and $22 \%$ aqueous $\mathrm{CsCl}$ solutions with benzyl alcohol. In this case, the horizontal axis represents the difference between 
the experimental density ratio and the critical density ratio obtained from simulations $\rho_{2} / \rho_{\text {lexp }}-\rho_{2} / \rho_{\text {lcrit(sim) }}$. As before, the uncertainty in the experimental density ratio is \pm 0.003 . The uncertainty in the predicted critical density ratio from simulations, shown as the shaded region in Figure $8 b$, is \pm 0.015 . The uncertainty for the simulations is slightly larger than that of the zero Bond number prediction owing to the limited ability of Surface Evolver to resolve the critical value. The numerical gap in the density ratios of the nearest observed energy maximum and minimum configurations is approximately $0.003-0.004$ in our Surface Evolver simulations. In Figure 8b, the experimentally realized transition from an energy maximum to an energy minimum overlaps well with the shaded region, demonstrating that the experimental results agree well with the simulations independent of the Bond number of the bottom drop. Although within uncertainty, it is noted that the critical density ratio difference tends to cluster at the positive edge of the shaded region, hinting that the spherical cap shape perturbations used in the simulations may yield a small systematic offset as compared with the experiments using pipettes to realize the perturbations. Further investigation of this possible systematic offset is beyond the scope of this paper and would require a more general stability analysis.

Similar experiments to examine the transition from energy minimum to energy maximum could also be performed using the phase-separated mixture of butanol and aqueous $\mathrm{CsCl}$ solutions. Besides the advantage of the low vapor pressure of benzyl alcohol, which minimizes evaporation, benzyl alcohol also has a density greater than water. For mixtures of butanol and aqueous $\mathrm{CsCl}$ solutions, increasing the concentration of $\mathrm{CsCl}$ causes the experimental density ratio to monotonically decrease; therefore the axisymmetric configuration will be more stable as 
the $\mathrm{CsCl}$ concentration increases. In contrast, the density ratio obtained from the mixtures of benzyl alcohol and aqueous $\mathrm{CsCl}$ solutions first increases to approximately 1 and then decreases to about 0.826 , indicating that the axisymmetric configuration first becomes less stable and then more stable as the concentration of $\mathrm{CsCl}$ increases. Thus the benzyl alcohol mixtures permit the stable-to-unstable transition to be realized more easily.

Figure 8 shows that the zero Bond number analysis, the Surface Evolver simulations, and the experiments all agree well in the observed and predicted transition from energy minimum to energy maximum compound drop configurations. At high Bond numbers, both the experiments and the Surface Evolver simulations indicate that the critical density ratio deviates from that obtained in the zero Bond number limit by approximately $3-4 \%$. Perturbing the compound sessile drop using a pipette tip is more likely to represent a generic perturbation in which axisymmetry is broken, and interface shapes are not restricted to either spherical caps or Laplacian shapes. Nevertheless, good agreement is found when comparing the stability criterion obtained for each of the three types of perturbations.

\section{Conclusions}

In this paper, we have determined seven dimensionless parameters that govern the shape of an axisymmetric compound sessile drop using the Laplace equations. Following a Laplacian shape perturbation, a stability criterion for the axisymmetric configuration is determined in the zero Bond number limit. This stability criterion is verified using both Surface Evolver simulations and experiments performed with both high and low interfacial tension systems.

For an axisymmetric compound drop ${ }^{11,12}$ to be stable, the density ratio must be smaller than 
a critical density ratio that depends on the surface and interfacial tensions, and the drop volumes. The critical density ratio is small for a high interfacial tension system, therefore stable axisymmetric configurations are rarely observed for organic/inorganic compound sessile drops in air. The critical density ratio is much larger for low interfacial tension systems ${ }^{42-45}$, allowing the possibility for experimental realization of stable configurations. We successfully observed stable axisymmetric compound sessile drops using a low interfacial tension system obtained from phase-separated mixtures of benzyl alcohol and aqueous $\mathrm{CsCl}$ solutions ${ }^{34,38}$. The low interfacial tension systems show a transition from energy minimum to energy maximum by varying the $\mathrm{CsCl}$ concentration, which varies the density ratio without significantly changing the surface and interfacial tensions. The critical condition for transition between the stable and unstable axisymmetric configurations agrees closely in the small Bond number cases for all three approaches: the zero Bond number analysis, the Surface Evolver simulations, and the experiments. The simulations and experiments exhibit a small deviation from the zero Bond number predictions at larger Bond numbers.

The ability to form a stable axisymmetric compound sessile drop may be useful in designing liquid lens systems for soft optics. Interestingly, the stability analysis predicts that a more dense drop resting on top of a less dense drop is never stable, even though a more dense drop is observed to float on top of a less dense bulk fluid using the low interfacial tension system obtained from phase separated mixtures of benzyl alcohol and aqueous $\mathrm{CsCl}$ solutions. Further investigation of this low interfacial tension system may provide a better understanding of the flotation mechanism of dense objects through the distortion of surfaces. 


\section{ACKNOWLEDGEMENTS}

This work is supported by the National Science Foundation (Grant No. CBET-1264552) and the Physics Department at Carnegie Mellon University.

\section{Appendix}

Table I. Densities, surface and interfacial tensions of dodecane and aqueous $\mathrm{CsCl}$ solutions.

\begin{tabular}{lccc}
\hline Fluid & Density $(\mathrm{g} / \mathrm{ml})$ & $\begin{array}{c}\text { Surface tension } \\
(\mathrm{mN} / \mathrm{m})\end{array}$ & $\begin{array}{c}\text { Interfacial tension } \\
\text { with dodecane } \\
(\mathrm{mN} / \mathrm{m})\end{array}$ \\
\hline Water & $0.9946 \pm 0.0032^{*}$ & $72.4 \pm 0.5$ & $52.2 \pm 0.3$ \\
$20 \%$ w/w CsCl & $1.1677 \pm 0.0035$ & $73.5 \pm 0.4$ & $50.1 \pm 0.5$ \\
$40 \%$ w/w CsCl & $1.4136 \pm 0.0027$ & $74.1 \pm 0.5$ & $53.9 \pm 0.3$ \\
$60 \%$ w/w CsCl & $1.7780 \pm 0.0023$ & $75.1 \pm 0.6$ & $58.3 \pm 0.4$ \\
Dodecane & $0.7431 \pm 0.0035$ & $25.3 \pm 0.4$ & - \\
\hline
\end{tabular}


Table II. Density of each liquid component collected from the phase-separated mixture of benzyl alcohol and aqueous $\mathrm{CsCl}$ solution with similar volumes.

\begin{tabular}{lcc}
\hline Liquid pair & Water rare phase $(\mathrm{g} / \mathrm{ml})$ & Water rich phase $(\mathrm{g} / \mathrm{ml})$ \\
\hline Benzyl-OH \& water & $1.0344 \pm 0.0025$ & $0.9968 \pm 0.0034$ \\
Benzyl-OH \& 6\%w/w CsCl & $1.0386 \pm 0.0021$ & $1.0470 \pm 0.0022$ \\
Benzyl-OH \& 10\%w/w CsCl & $1.0389 \pm 0.0021$ & $1.0869 \pm 0.0023$ \\
Benzyl-OH \& 14\%w/w CsCl & $1.0402 \pm 0.0020$ & $1.1253 \pm 0.0023$ \\
Benzyl-OH \& 18\%w/w CsCl & $1.0404 \pm 0.0021$ & $1.1658 \pm 0.0027$ \\
Benzyl-OH \& 22\%w/w CsCl & $1.0402 \pm 0.0021$ & $1.2102 \pm 0.0036$ \\
Benzyl-OH \& 26\%w/w CsCl & $1.0422 \pm 0.0020$ & $1.2613 \pm 0.0037$ \\
\hline
\end{tabular}


Table III. Surface and interfacial tension measurements for the two liquid phases obtained from phase separated mixtures of benzyl alcohol and aqueous $\mathrm{CsCl}$ solutions with similar volumes.

\begin{tabular}{lccc}
\hline Liquid pair & $\begin{array}{c}\text { Surface tension of } \\
\text { water rare phase } \\
(\mathrm{mN} / \mathrm{m})\end{array}$ & $\begin{array}{c}\text { Surface tension of } \\
\text { water rich phase } \\
(\mathrm{mN} / \mathrm{m})\end{array}$ & $\begin{array}{c}\text { Interfacial } \\
\text { tension of two } \\
\text { phases }(\mathrm{mN} / \mathrm{m})\end{array}$ \\
\hline Benzyl-OH \& water & $39.5 \pm 0.5$ & $41.5 \pm 0.6$ & $3.0 \pm 0.3$ \\
Benzyl-OH \& 6\%w/w CsCl & $38.6 \pm 0.4$ & $40.2 \pm 0.5$ & $5.0 \pm 0.3$ \\
Benzyl-OH \& 10\%w/w CsCl & $38.9 \pm 0.5$ & $40.5 \pm 0.5$ & $5.1 \pm 0.2$ \\
Benzyl-OH \& 14\%w/w CsCl & $38.6 \pm 0.5$ & $41.0 \pm 0.3$ & $5.3 \pm 0.3$ \\
Benzyl-OH \& 18\%w/w CsCl & $39.0 \pm 0.4$ & $41.6 \pm 0.4$ & $5.8 \pm 0.3$ \\
Benzyl-OH \& 22\%w/w CsCl & $39.5 \pm 0.3$ & $41.9 \pm 0.3$ & $6.2 \pm 0.2$ \\
Benzyl-OH \& 26\%w/w CsCl & $39.3 \pm 0.3$ & $42.3 \pm 0.5$ & $6.6 \pm 0.4$ \\
\hline
\end{tabular}




\section{References}

1 J.B. Keller, "Surface tension force on a partly submerged body," Phys. Fluids 10, 3009-3010 (1998).

$2 \quad$ X. Gao and L. Jiang, "Biophysics: water-repellent legs of water striders.," Nature 432, 36 (2004).

3 C.W. Extrand and S.I. Moon, "Using the flotation of a single sphere to measure and model capillary forces.," Langmuir 25(11), 6239-44 (2009).

I. Ivanov, P.A. Kralchevsky, and A.D. Nikolov, "Film and line tension effects on the attachment of particles to an interface," J. Colloid Interface Sci. 112(1), 97 (1986).

P. Pujado and L. Scriven, "Sessile lenticular configurations: translationally and rotationally symmetric lenses,” J. Colloid Interface Sci. 40, (1972).

L. Boruvka and A.W. Neumann, "Generalization of the classical theory of capillarity," J. Chem. Phys. 66, 5464 (1977).

R. Aveyard and J. Clint, "Liquid lenses at fluid/fluid interfaces," J. Chem. Soc. Faraday Trans. 1(4), 1397-1403 (1997).

C.M. Phan, B. Allen, L.B. Peters, T.N. Le, and M.O. Tade, "Can water float on oil?,” Langmuir 28(10), 4609-13 (2012).

C.M. Phan, "Stability of a floating water droplet on an oil surface.," Langmuir 30(3), 768-73 (2014).

D. Chatain, P. Wynblatt, M. De Ruijter, J. De Conninck, and C. Carter, "Shape of two-phase drops in the presence of perfect wetting," Acta Mater. 47, 3049-3056 (1999).

L. Mahadevan, M. Adda-Bedia, and Y. Pomeau, "Four-phase merging in sessile compound drops," J. Fluid Mech. 451, 411-420 (2002).

M.J. Neeson, R.F. Tabor, F. Grieser, R.R. Dagastine, and D.Y.C. Chan, "Compound sessile drops," Soft Matter 8(43), 11042 (2012).

A. Abate and D. Weitz, "High-order multiple emulsions formed in poly(dimethylsiloxane) microfluidics.," Small 5(18), 2030-2 (2009).

M. Seo, C. Paquet, Z. Nie, S. Xu, and E. Kumacheva, "Microfluidic consecutive flow-focusing droplet generators," Soft Matter 3(8), 986 (2007).

M. Ben Said, M. Selzer, B. Nestler, and D. Braun, "A phase-field approach for wetting phenomena of multiphase droplets on solid surfaces," Langmuir (2014). 
S. Torza and S. Mason, "Three-phase interactions in shear and electrical fields," J. Colloid Interface Sci. 33(1), 67 (1970).

17

R. Johnson and S. Sadhal, "Fluid mechanics of compound multiphase drops and bubbles," Annu. Rev. Fluid Mech. 17, 289-320 (1985).

K. Brakke, “The surface evolver,” Exp. Math. 1(2), 141-165 (1992).

K.A. Brakke, The Surface Evolver and the Stability of Liquid Surfaces, Philos. Trans. R. Soc. A Math. Phys. Eng. Sci. 354, 2143-2157 (1996).

J. Berthier and K.A. Brakke, The Physics of Microdroplets (John Wiley \& Sons, Hoboken, NJ, 2012).

S.G. Krantz and H.R. Parks, The Geometry of Domains in Space (Springer, 1999).

A. Chesters, "An analytical solution for the profile and volume of a small drop or bubble symmetrical about a vertical axis,” J. Fluid Mech. 81, 609-624 (1977).

P.G. Smith and T.G.M. Van De Ven, Profiles of slightly deformed axisymmetric drops, J. Colloid Interface Sci. 97, 1-8 (1984).

S. Rienstra, "The shape of a sessile drop for small and large surface tension," J. Eng. Math. 193-202 (1990).

V. Lubarda, "Mechanics of a liquid drop deposited on a solid substrate," Soft Matter 10288-10297 (2012).

J.F. Padday and A.R. Pitt, The Stability of Axisymmetric Menisci, Philos. Trans. R. Soc. A Math. Phys. Eng. Sci. 275, 489-528 (1973).

J.H. Snoeijer and B. Andreotti, “A microscopic view on contact angle selection,” Phys. Fluids 20(5), 057101 (2008).

S.R. Majumdar and D.H. Michael, The Equilibrium and Stability of Two Dimensional Pendent Drops, Proc. R. Soc. A Math. Phys. Eng. Sci. 351, 89-115 (1976).

P.T. Sumesh and R. Govindarajan, "The possible equilibrium shapes of static pendant drops.," J. Chem. Phys. 133(14), 144707 (2010).

L. Landau and E. Lifshitz, Mechanics, Vol. 1 of Course of Theoretical Physics, 3rd Editio (Butterworth-Heinemann, 1976).

H. Goldstein, P.C. Poole, and J.L. Safko, Classical Mechanics (Pearson, 2002). 
A.W. Adamson and A.P. Gast, Physical Chemistry of Surfaces, 6th ed. (Wiley, New York, 1997).

A. Demond and A. Lindner, "Estimation of interfacial tension between organic liquids and water," Environ. Sci. Technol. 27(12), 2318-2331 (1993).

J. Gibbs, The Scientific Papers of J Willard Gibbs. Vol 1, Thermodynamics (Dover, New York, 1961).

J. Guzowski, P.M. Korczyk, S. Jakiela, and P. Garstecki, The structure and stability of multiple micro-droplets, Soft Matter 8, 7269 (2012).

W.. Haynes, CRC Handbook of Chemistry and Physics, 81st ed. (Taylor \& Francis group, New York, 2000).

Y. Zhang, S.D. Oberdick, E.R. Swanson, S.L. Anna, and S. Garoff, "Gravity driven current during the coalescence of two sessile drops," Phys. Fluids 27(2), 022101 (2015).

R. David, S.M. Dobson, Z. Tavassoli, M.G. Cabezas, and A.W. Neumann, "Investigation of the Neumann triangle for dodecane liquid lenses on water," Colloids Surfaces A Physicochem. Eng. Asp. 333(1-3), 12-18 (2009).

H.-J. Butt, K. Graf, and M. Kappl, Physics and Chemistry of Interfaces (Wiley-VCH Verlag GmbH \& Co. KGaA, Weinheim, 2003).

D.J. Donahue, F.E. Bartell, U. Michigan, and A. Arbor, "The boundary tension at water-organic liquid interfaces," J. Phys. Chem. 56, 480-484 (1952).

D.G.A.L. Aarts, M. Schmidt, and H.N.W. Lekkerkerker, "Direct visual observation of thermal capillary waves.," Science 304, 847-850 (2004).

H.N.W. Lekkerkerker et al., "Life at ultralow interfacial tension: Wetting, waves and droplets in demixed colloid-polymer mixtures,” Eur. Phys. J. B 64, 341-347 (2008).

M.C. van Loosdrecht, J. Lyklema, W. Norde, G. Schraa, and A.J. Zehnder, "The role of bacterial cell wall hydrophobicity in adhesion.," Appl. Environ. Microbiol. 53(8), 1893-1897 (1987).

Y. Liu, R. Lipowsky, and R. Dimova, "Concentration dependence of the interfacial tension for aqueous two-phase polymer solutions of dextran and polyethylene glycol," Langmuir 28(8), 3831-3839 (2012).

T.M. Duncan and J.A. Reimer, Chemical Engineering Design and Analysis: An Introduction (Cambridge University Press, Cambridge, 1998).

E.B. Munday, J.C. Mullins, and D.D. Edie, "Vapor pressure data for toluene, 1-pentanol, 1-butanol, water, and 1-propanol and for the water and 1-propanol system from 273.15 to 323.15," J. Chem. Eng. Data 25, 191-194 (1980). 
D. Ambrose and N.B. Ghiassee, "Vapour pressures, critical temperatures, and critical pressures of benzyl alcohol, octan-2-ol, and 2-ethylhexan-1-ol,” J. Chem. Thermodyn. 22, 307-311 (1990). 


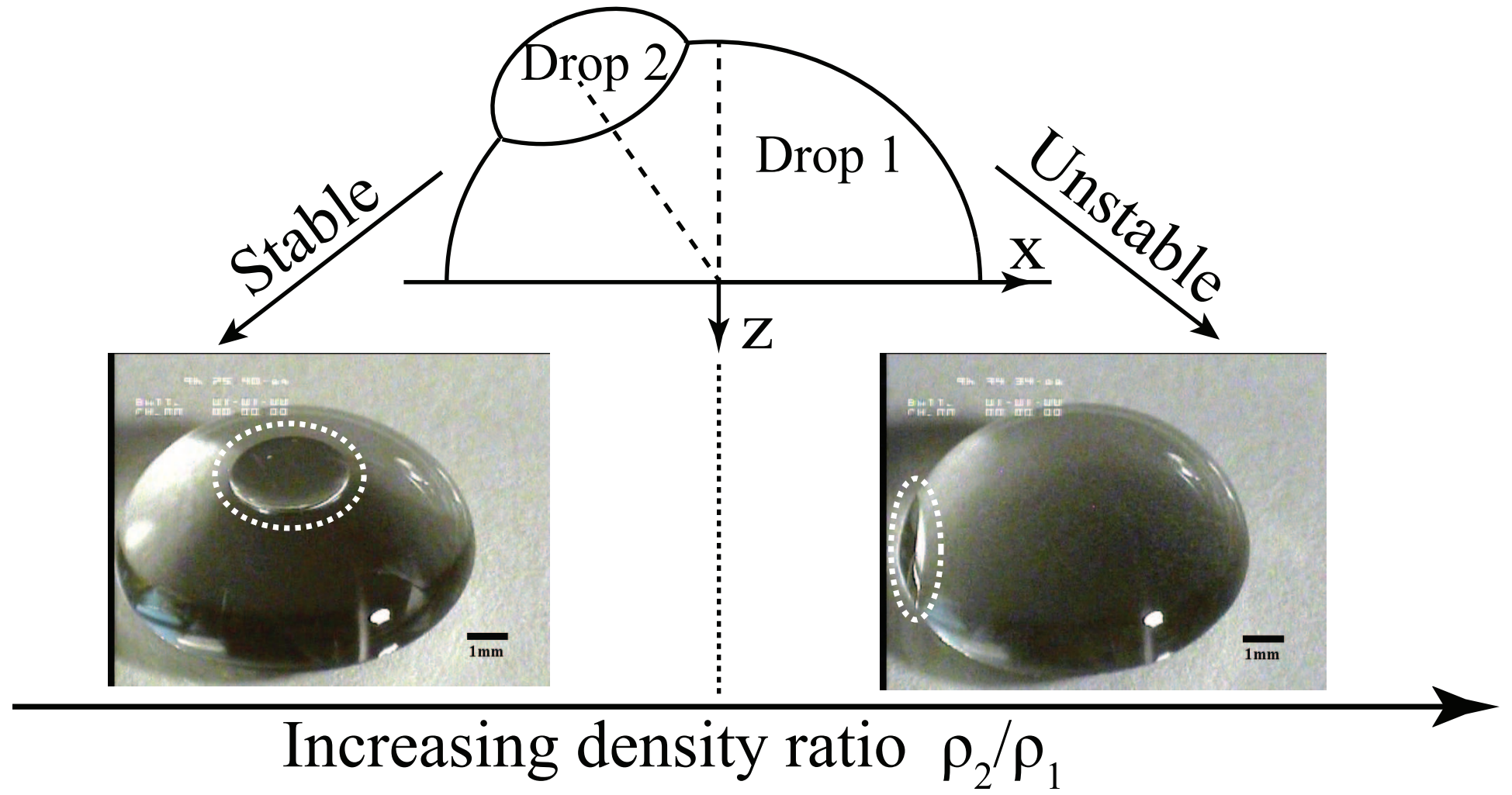

Graphical Abstract 